\title{
Bases in which some numbers have exactly two expansions
}

\author{
Vilmos Komornik \\ Département de mathématique, Université de Strasbourg, 7 rue René Descartes, 67084 Strasbourg Cedex, France \\ Derong Kong* \\ Mathematical Institute, University of Leiden, PO Box 9512, 2300 RA Leiden, The Netherlands
}

\begin{abstract}
In this paper we answer several questions raised by Sidorov on the set $\mathcal{B}_{2}$ of bases in which there exist numbers with exactly two expansions. In particular, we prove that the set $\mathcal{B}_{2}$ is closed, and it contains both infinitely many isolated and accumulation points in $\left(1, q_{K L}\right)$, where $q_{K L} \approx 1.78723$ is the Komornik-Loreti constant. Consequently we show that the second smallest element of $\mathcal{B}_{2}$ is the smallest accumulation point of $\mathcal{B}_{2}$. We also investigate the higher order derived sets of $\mathcal{B}_{2}$. Finally, we prove that there exists a $\delta>0$ such that

$$
\operatorname{dim}_{H}\left(\mathcal{B}_{2} \cap\left(q_{K L}, q_{K L}+\delta\right)\right)<1,
$$
\end{abstract}

where $\operatorname{dim}_{H}$ denotes the Hausdorff dimension.

Keywords: Non-integer bases, two expansions, derived sets, accumulation points, unique expansion, Hausdorff dimension 2010 MSC: Primary: 11A63, Secondary: 37F20, 37B10

\section{Introduction}

In this paper we consider expansions

$$
\left(\left(c_{i}\right)\right)_{q}:=\sum_{i=1}^{\infty} \frac{c_{i}}{q^{i}}=x
$$

over the alphabet $\{0,1\}$ in some base $q>1$. The sequence $\left(c_{i}\right)=c_{1} c_{2} \cdots$ is called a $q$-expansion of $x$. Such an expansion may exist only if $x \in I_{q}:=[0,1 /(q-1)]$.

If $q>2$, then every expansion is unique by an elementary argument, but not every $x \in I_{q}$ has an expansion. If $q \in(1,2]$, then every $x \in I_{q}$ has at least one expansion. For example, in the well-known integer base case $q=2$ all numbers have a unique expansion, except the dyadic rational numbers that have two. Henceforth we assume that $q \in(1,2]$.

Expansions in non-integer bases $q \in(1,2)$ are more complicated than that in the integer base $q=2$. Erdôs et al. discovered in [10, 11, 12] that for any $k \in \mathbb{N} \cup\left\{\aleph_{0}\right\}$ there exist $q \in(1,2)$ and $x \in I_{q}$ such that $x$ has precisely $k$ different $q$-expansions. Furthermore, in case $q \in(1, \varphi)$, where $\varphi \approx 1.61803$ denotes the Golden Ratio, every $x \in(0,1 /(q-1))$ has a continuum of $q$-expansions.

\footnotetext{
${ }^{*}$ Corresponding author

Email addresses: komornik@math.unistra.fr (Vilmos Komornik), d.kong@math.leidenuniv.nl (Derong Kong)
} 
Later, Sidorov proved in [26] that for any $q \in(1,2)$ Lebesgue almost every $x \in I_{q}$ has a continuum of $q$-expansions (see also [5]).

Many works have been devoted to unique expansions. Komornik and Loreti proved in [19] that there is a smallest base $q_{K L} \approx 1.78723$ in which $x=1$ has a unique expansion. Subsequently, Glendinning and Sidorov discovered that the Komornik-Loreti constant $q_{K L}$ plays an important role in describing the size of the univoque sets

$$
\mathcal{U}_{q}:=\left\{x \in I_{q}: x \text { has a unique } q \text {-expansion }\right\}, \quad q \in(1,2] .
$$

They proved in [14] the following interesting results:

- if $1<q \leq \varphi$, then $\mathcal{U}_{q}=\{0,1 /(q-1)\}$;

- if $\varphi<q<q_{K L}$, then $\mathcal{U}_{q}$ is countably infinite;

- if $q=q_{K L}$, then $\mathcal{U}_{q}$ is uncountable and has zero Hausdorff dimension;

- if $q_{K L}<q \leq 2$, then $\operatorname{dim}_{H} \mathcal{U}_{q}>0$, where $\operatorname{dim}_{H}$ denotes the Hausdorff dimension.

We point out that $\mathcal{U}_{q}$ has a fractal structure for $q \in\left(q_{K L}, 2\right)$. The authors and Li proved in [18] that the function $q \mapsto \operatorname{dim}_{H} \mathcal{U}_{q}$ has a Devil's staircase behavior.

Based on the classification of the bases $q \in(1,2]$ by Komornik and Loreti [20], the topology of the univoque sets $\mathcal{U}_{q}$ was studied by de Vries and Komornik in [7]. They proved among others that $\mathcal{U}_{q}$ is closed if and only if $q \notin \overline{\mathcal{U}}$, where $\overline{\mathcal{U}}$ is the topological closure of

$$
\mathcal{U}:=\left\{q \in(1,2]: 1 \in \mathcal{U}_{q}\right\} .
$$

In other words, $\mathcal{U}$ is the set of univoque bases $q \in(1,2]$ for which 1 has a unique expansion. An element of $\mathcal{U}$ is called a univoque base. As mentioned above, the smallest univoque base is $q_{K L}$.

The set $\mathcal{U}$ itself is also of general interest. Erdôs et al. proved in [11] that $\mathcal{U}$ is a Lebesgue null set of first category. Later, Daróczy and Kátai proved in [6] that $\mathcal{U}$ has full Hausdorff dimension. The topological structure of $\mathcal{U}$ was clarified in [20]. In particular, the authors proved that $\overline{\mathcal{U}}$ is a Cantor set, and $\overline{\mathcal{U}} \backslash \mathcal{U}$ is a countable and dense subset of $\overline{\mathcal{U}}$. Recently, Bonanno et al. investigated in [4] the connections between $\mathcal{U}, \alpha$-continued fractions, unimodal maps and even the external rays of the Mandelbrot set.

For more information on the sets $\mathcal{U}_{q}$ and $\mathcal{U}$ we refer to the survey paper [15] and the book chapter [8].

Sidorov initiated in [27] the study of the sets

$$
\mathcal{B}_{k}:=\left\{q \in(1,2]: \exists x \in I_{q} \text { having precisely } k \text { different } q \text {-expansions }\right\}
$$

for $k=2,3, \ldots$ and $k=\aleph_{0}$. In particular, he has obtained the following important results for the set $\mathcal{B}_{2}$.

Theorem 1.1 (Sidorov, 2009).

(i) $q \in \mathcal{B}_{2} \Longleftrightarrow 1 \in \mathcal{U}_{q}-\mathcal{U}_{q}$;

(ii) $\mathcal{U} \subset \mathcal{B}_{2}$;

(iii) $\left[\varphi_{3}, 2\right] \subset \mathcal{B}_{2}$, where $\varphi_{3} \approx 1.83929$ denotes the Tribonacci number, i.e., the positive zero of $q^{3}-q^{2}-q-1$;

(iv) the smallest two elements of $\mathcal{B}_{2}$ are

$$
q_{s} \approx 1.71064 \text { and } q_{f} \approx 1.75488 \text {, }
$$

the positive zeros of the polynomials $q^{4}-2 q^{2}-q-1$ and $q^{3}-2 q^{2}+q-1$ respectively. 
Theorem 1.1 (ii) was also contained in [7, Theorem 1.3].

Until now very little is known about the sets $\mathcal{B}_{k}$. Sidorov proved in [27] that $\mathcal{B}_{k}$ contains a left neighborhood of 2 for each $k \geq 2$. Baker and Sidorov proved in [3] that the second smallest element $q_{f}$ of $\mathcal{B}_{2}$ is the smallest element of $\mathcal{B}_{k}$ for each $k \geq 3$. It follows from the results of Erdôs et al. [10, 11] that the Golden Ratio $\varphi$ is the smallest element of $\mathcal{B}_{\aleph_{0}}$. Recently, Baker proved in 2] that $\mathcal{B}_{\aleph_{0}}$ has a second smallest element which is strictly smaller than the smallest element $q_{s}$ of $\mathcal{B}_{2}$. Based on his work Zou and Kong proved in [29] that $\mathcal{B}_{\aleph_{0}}$ is not closed.

The purpose of this paper is to continue the investigations on the set $\mathcal{B}_{2}$. We answer in particular the following questions of Sidorov [27]:

Q1. Is $\mathcal{B}_{2}$ a closed set?

Q2. Is $\mathcal{B}_{2} \cap\left(1, q_{K L}\right)$ a discrete set?

Q3. Is it true that

$$
\operatorname{dim}_{H}\left(\mathcal{B}_{2} \cap\left(q_{K L}, q_{K L}+\delta\right)\right)<1
$$

for some $\delta>0$ ?

Some ideas of this paper might be useful for the future study of $\mathcal{B}_{k}$ with $k \geq 3$.

Motivated by the work of de Vries and Komornik [7] (see also [16]) we introduce the sets

$$
\mathcal{V}_{q}:=\left\{x \in I_{q}: x \text { has at most one doubly infinite } q \text {-expansion }\right\}, \quad q \in(1,2] .
$$

Here and in the sequel an expansion is called infinite if it does not end with $10^{\infty}$ (it does not have a last one digit), and doubly infinite if it does not end with $01^{\infty}$ or $10^{\infty}$ (it has neither a last one digit, nor a last zero digit).

Remark 1.2. If $q$ is not an integer, then each $x \in I_{q}$ has a doubly infinite $q$-expansion, namely its quasi-greedy expansion. See also [28].

We recall from [7] that $\mathcal{V}_{q}$ is closed, and

$$
\mathcal{U}_{q} \subseteq \overline{\mathcal{U}_{q}} \subseteq \mathcal{V}_{q}
$$

for all $q \in(1,2]$, where $\overline{\mathcal{U}_{q}}$ denotes the topological closure of $\mathcal{U}_{q}$.

Using these sets we add two new characterizations of $\mathcal{B}_{2}$ to Theorem 1.1(i):

Theorem 1.3. The following conditions are equivalent:

(i) $q \in \mathcal{B}_{2}$;

(ii) $1 \in \mathcal{U}_{q}-\mathcal{U}_{q}$;

(iii) $1 \in \overline{\mathcal{U}_{q}}-\overline{\mathcal{U}_{q}}$;

(iv) $1 \in \mathcal{V}_{q}-\mathcal{V}_{q}$ and $q \neq \varphi$.

In order to state our next results we recall from [20] and [16] the notation

$$
\mathcal{V}:=\left\{q \in(1,2]: 1 \in \mathcal{V}_{q}\right\} ;
$$

this is the set of bases $q \in(1,2]$ in which 1 has a unique doubly infinite $q$-expansion.

Remark 1.4. The number $x=1$ always has a doubly infinite $q$-expansion, namely its quasi-greedy expansion, even in integer bases. 
We recall from [20] that $\mathcal{V}$ is closed, and that

$$
\mathcal{U} \subset \overline{\mathcal{U}} \subset \mathcal{V} .
$$

The smallest element of $\mathcal{V}$ is the Golden Ratio $\varphi$, while the smallest element of $\mathcal{U}$ (and $\overline{\mathcal{U}}$ ) is $q_{K L}$. Furthermore, the difference set $\overline{\mathcal{U}} \backslash \mathcal{U}$ is countably infinite and dense in $\overline{\mathcal{U}}$, and the difference set $\mathcal{V} \backslash \overline{\mathcal{U}}$ is discrete, countably infinite and dense in $\mathcal{V}$.

We recall from [7, 9] that $(1,2] \backslash \overline{\mathcal{U}}=(1,2) \backslash \overline{\mathcal{U}}$ is the disjoint union of its connected components $\left(q_{0}, q_{0}^{*}\right)$, where $q_{0}$ runs over $\{1\} \cup(\overline{\mathcal{U}} \backslash \mathcal{U})$ and $q_{0}^{*}$ runs over a proper subset $\mathcal{U}^{*}$ of $\mathcal{U}$. Since the Komornik-Loreti constant $q_{K L}$ is the smallest element of $\overline{\mathcal{U}}$, the first connected component is $\left(1, q_{K L}\right)$.

We recall that each left endpoint $q_{0}$ is an algebraic integer, and each right endpoint $q_{0}^{*}$, called a de Vries-Komornik number, is a transcendental number; see Kong and Li [21].

We also recall that for each component, $\mathcal{V} \cap\left(q_{0}, q_{0}^{*}\right)$ is formed by an increasing sequence $q_{1}<q_{2}<\cdots$ of algebraic integers, converging to $q_{0}^{*}$. For example, the first two elements of $\mathcal{V}$ in the first connected component $\left(1, q_{K L}\right)$ are the Golden Ratio $\varphi$ and the second smallest element $q_{f}$ of $\mathcal{B}_{2}$; see also Example 2.6 below.

Now we state our basic results on $\mathcal{B}_{2}$ :

\section{Theorem 1.5.}

(i) The set $\mathcal{B}_{2}$ is compact.

(ii) $\mathcal{V} \backslash\{\varphi\} \subset \mathcal{B}_{2}$.

(iii) Each element of $\mathcal{V} \backslash\{\varphi\}$ is an accumulation point of $\mathcal{B}_{2}$. Hence $\mathcal{B}_{2}$ has infinitely many accumulation points in each connected component $\left(q_{0}, q_{0}^{*}\right)$ of $(1,2) \backslash \overline{\mathcal{U}}$.

(iv) The smallest accumulation point of $\mathcal{B}_{2}$ is its second smallest element $q_{f}$.

(v) $\mathcal{B}_{2} \cap\left(1, q_{K L}\right)$ contains only algebraic integers, and hence it is countable.

(vi) $\mathcal{B}_{2}$ has infinitely many isolated points in $\left(1, q_{K L}\right)$, and they are dense in $\mathcal{B}_{2} \cap\left(1, q_{K L}\right)$.

\section{Remarks 1.6.}

- Theorem 1.5 (i) answers positively Sidorov's question Q1.

- Theorem 1.5 (ii) improves Theorem 1.1 (ii) because $\mathcal{U}$ (and even $\overline{\mathcal{U}}$ ) is a proper subset of $\mathcal{V} \backslash\{\varphi\}$.

- Since $\left(1, q_{K L}\right)$ is a connected component of $(1,2) \backslash \overline{\mathcal{U}}$, Theorem 1.5 (iii) answers negatively Sidorov's question Q2.

- Theorem 1.5 (iv) answers partially a question of Baker and Sidorov [3] about the smallest accumulation point of $\mathcal{B}_{k}$ for $k \geq 2$. We recall that the smallest accumulation point $q_{f}$ of $\mathcal{B}_{2}$ is the smallest element of $\mathcal{B}_{k}$ for all $k \geq 3$.

- Theorem[1.5 (v) strengthens a result of Sidorov [2\}] stating that $\mathcal{B}_{2} \cap\left(1, q_{K L}\right)$ contains only algebraic numbers.

In the following theorem we show that $\mathcal{B}_{2}$ contains infinitely many accumulation points of all finite orders in $\left(1, q_{K L}\right)$. For this we introduce the derived sets $\mathcal{B}_{2}^{(0)}, \mathcal{B}_{2}^{(1)}, \ldots$ by induction, setting $\mathcal{B}_{2}^{(0)}:=\mathcal{B}_{2}$, and then

$$
\mathcal{B}_{2}^{(j+1)}=\left\{q \in(1,2]: q \text { is an accumulation point of } \mathcal{B}_{2}^{(j)}\right\}
$$


for $j=0,1, \ldots$.

All these sets are compact by Theorem 1.5 (i) and a general property of derived sets. Since

$$
\mathcal{B}_{2}=\mathcal{B}_{2}^{(0)} \supseteq \mathcal{B}_{2}^{(1)} \supseteq \mathcal{B}_{2}^{(2)} \supseteq \cdots,
$$

the derived set

$$
\mathcal{B}_{2}^{(\infty)}:=\cap_{j=0}^{\infty} \mathcal{B}_{2}^{(j)}
$$

of infinite order is also well defined, non-empty and compact.

\section{Theorem 1.7.}

(i) $\overline{\mathcal{U}} \subset \mathcal{B}_{2}^{(\infty)}$.

(ii) $\mathcal{V} \backslash\{\varphi\} \subset \mathcal{B}_{2}^{(2)}$.

(iii) $\min \mathcal{B}_{2}^{(1)}=\min \mathcal{B}_{2}^{(2)}=q_{f}$.

(iv) All sets $\mathcal{B}_{2}, \mathcal{B}_{2}^{(1)}, \mathcal{B}_{2}^{(2)}, \ldots$ have infinitely many accumulation points in each connected component $\left(q_{0}, q_{0}^{*}\right)$ of $(1,2] \backslash \overline{\mathcal{U}}$.

(v) If $q_{1}<q_{2}<\cdots$ are the elements of $\mathcal{V} \cap\left(1, q_{K L}\right)$, then

$$
q_{j+1} \leq \min \mathcal{B}_{2}^{(2 j)}<q_{2 j+1} \quad \text { for all } j \geq 0,
$$

and hence

$$
\min \mathcal{B}_{2}^{(j)} \nearrow \min \mathcal{B}_{2}^{(\infty)}=q_{K L} \quad \text { as } \quad j \rightarrow \infty .
$$

(vi) For each $j=0,1, \ldots, \mathcal{B}_{2}^{(j)} \cap\left(1, q_{K L}\right)$ has infinitely many isolated points, and they are dense in $\mathcal{B}_{2}^{(j)} \cap\left(1, q_{K L}\right)$.

Remark 1.8. Theorem 1.7 (iv) provides a negative answer to the question Q2 even if we replace $\mathcal{B}_{2}$ by $\mathcal{B}_{2}^{(j)}$.

Our last result is related to the local dimension of $\mathcal{B}_{2}$.

Theorem 1.9. For any $q \in \mathcal{B}_{2}$ we have

$$
\lim _{\delta \rightarrow 0} \operatorname{dim}_{H}\left(\mathcal{B}_{2} \cap(q-\delta, q+\delta)\right) \leq 2 \operatorname{dim}_{H} \mathcal{U}_{q}
$$

\section{Remarks 1.10.}

- We recall from [18] that the dimension function $q \mapsto \operatorname{dim}_{H} \mathcal{U}_{q}$ is continuous, and vanishes at the Komornik-Loreti constant $q_{K L}$ (see also Lemma 2.4 below). Since $q_{K L} \in \mathcal{U} \subset \mathcal{B}_{2}$, by Theorem 1.5 ( $v$ ) and Theorem 1.9 there exists a $\delta>0$ such that

$$
\operatorname{dim}_{H}\left(\mathcal{B}_{2} \cap\left(q_{K L}, q_{K L}+\delta\right)\right)=\operatorname{dim}_{H}\left(\mathcal{B}_{2} \cap\left(q_{K L}-\delta, q_{K L}+\delta\right)\right)<1 .
$$

This answers affirmatively Sidorov's question Q3.

- A related result, recently obtained in [22], states that

$$
\operatorname{dim}_{H}\left(\mathcal{B}_{2} \cap\left(q_{K L}, q_{K L}+\delta\right)\right)>0 \text { for all } \delta>0 .
$$

The rest of the paper is arranged in the following way. In Section 2 we recall some results from unique expansions. Based on the properties of unique expansions we describe the set $\mathcal{B}_{2}$ in Section 3. In Section 4 we prove Theorem [1.3, and the first parts of Theorems 1.5] and 1.7 Section 5 is devoted to the detailed description of unique expansions. This important tool is extensively used in the rest of the paper. The remaining parts of Theorems 1.5 and 1.7 are proved in Sections 6 and 7 and the proof of Theorem 1.9 is given in Section 8 . In the final section 9 we formulate some open questions related to the results of this paper. 


\section{Unique expansions}

In this section we recall several results on unique expansions that will be used to prove our main theorems. Let $\{0,1\}^{\infty}$ be the set of sequences $\left(d_{i}\right)=d_{1} d_{2} \cdots$ with elements $d_{i} \in\{0,1\}$. Let $\sigma$ be the left shift on $\{0,1\}^{\infty}$ defined by $\sigma\left(\left(d_{i}\right)\right):=\left(d_{i+1}\right)$. Then $\left(\{0,1\}^{\infty}, \sigma\right)$ is a full shift. Accordingly, let $\{0,1\}^{*}$ denote the set of all finite strings of zeros and ones, called words, together with the empty word denoted by $\epsilon$. For a word $\mathbf{c}=c_{1} \cdots c_{m} \in\{0,1\}^{*}$ we denote by $\mathbf{c}^{k}=\left(c_{1} \cdots c_{m}\right)^{k}$ the $k$-fold concatenation of $\mathbf{c}$ with itself, and by $\mathbf{c}^{\infty}=\left(c_{1} \cdots c_{m}\right)^{\infty}$ the periodic sequence with period block $\mathbf{c}$. For a word $\mathbf{c}=c_{1} \cdots c_{m}$ we set

$$
\mathbf{c}^{+}:=c_{1} \cdots c_{m-1}\left(c_{m}+1\right)
$$

if $c_{m}=0$, and

$$
\mathbf{c}^{-}:=c_{1} \cdots c_{m-1}\left(c_{m}-1\right)
$$

if $c_{m}=1$. The reflection of a word $\mathbf{c}=c_{1} \cdots c_{m}$ is defined by the formula

$$
\overline{\mathbf{c}}:=\left(1-c_{1}\right) \cdots\left(1-c_{m}\right),
$$

and the reflection of a sequence $\left(d_{i}\right) \in\{0,1\}^{\infty}$ is defined by

$$
\overline{\left(d_{i}\right)}=\left(1-d_{1}\right)\left(1-d_{2}\right) \cdots \text {. }
$$

In this paper we use lexicographical orderings $<, \leq,>$ and $\geq$ between sequences and words. Given two sequences $\left(c_{i}\right),\left(d_{i}\right) \in\{0,1\}^{\infty}$ we write $\left(c_{i}\right)<\left(d_{i}\right)$ or $\left(d_{i}\right)>\left(c_{i}\right)$ if there exists an $n \in \mathbb{N}$ such that $c_{1} \cdots c_{n-1}=d_{1} \cdots d_{n-1}$ and $c_{n}<d_{n}$. Furthermore, we write $\left(c_{i}\right) \leq\left(d_{i}\right)$ or $\left(d_{i}\right) \geq\left(c_{i}\right)$ if $\left(c_{i}\right)<\left(d_{i}\right)$ or $\left(c_{i}\right)=\left(d_{i}\right)$. Finally, for two words $\mathbf{u}, \mathbf{v} \in\{0,1\}^{*}$ we write $\mathbf{u}<\mathbf{v}$ or $\mathbf{v}>\mathbf{u}$ if $\mathbf{u} 0^{\infty}<\mathbf{v} 0^{\infty}$.

Now we recall some notations and results from unique expansions. Given a base $q \in(1,2]$ we denote by $\beta(q)=\left(\beta_{i}(q)\right)$ the lexicographically largest (called greedy) expansion of 1 in base $q$. Accordingly, we denote by

$$
\alpha(q)=\alpha_{1}(q) \alpha_{2}(q) \cdots
$$

the lexicographically largest infinite (called quasi-greedy) expansion of 1 in base q. Here an expansion is infinite if it has infinitely many one digits. If $\beta(q)=\beta_{1}(q) \cdots \beta_{n}(q) 0^{\infty}$ with $\beta_{n}(q)=1$, then $\alpha(q)$ is periodic and $\alpha(q)=\left(\beta_{1}(q) \cdots \beta_{n}(q)^{-}\right)^{\infty}$.

The following Parry type property (see [25]) of $\alpha(q)$ was given in [1]:

Lemma 2.1. The map $q \mapsto \alpha(q)$ is a strictly increasing bijection from $(1,2]$ onto the set of all infinite sequences $\left(a_{i}\right) \in\{0,1\}^{\infty}$ satisfying

$$
a_{n+1} a_{n+2} \cdots \leq a_{1} a_{2} \cdots \text { whenever } a_{n}=0 .
$$

We recall that $\mathcal{U}_{q}$ is the set of $x \in[0,1 /(q-1)]$ having a unique $q$-expansion. We denote by $\mathcal{U}_{q}^{\prime}$ the set of expansions of all $x \in \mathcal{U}_{q}$. The following lexicographical characterization is a simple variant of another one given by Erdős et al. [10].

Lemma 2.2. Let $q \in(1,2]$. Then $\left(c_{i}\right) \in \mathcal{U}_{q}^{\prime}$ if and only if

$$
\begin{aligned}
& c_{n+1} c_{n+2} \cdots<\alpha(q) \quad \text { whenever } \quad c_{n}=0, \\
& \overline{c_{n+1} c_{n+2} \cdots<\alpha(q)} \text { whenever } \quad c_{n}=1 .
\end{aligned}
$$

Lemmas 2.1 and 2.2 imply that the set-valued map $q \mapsto \mathcal{U}_{q}^{\prime}$ is increasing, i.e., $\mathcal{U}_{p}^{\prime} \subseteq \mathcal{U}_{q}^{\prime}$ if $p<q$.

We recall that $\mathcal{V}_{q}$ is the set of $x \in I_{q}$ having at most one doubly infinite $q$-expansion. The following lexicographical characterization of $\mathcal{V}_{q}$ was given in [7]. 
Lemma 2.3. Let $q \in(1,2]$. Then $x \in \mathcal{V}_{q}$ if and only if $x$ has a q-expansion $\left(c_{i}\right)$ satisfying

$$
\begin{aligned}
& c_{n+1} c_{n+2} \cdots \leq \alpha(q) \quad \text { whenever } \quad c_{n}=0 \\
& \overline{c_{n+1} c_{n+2} \cdots \leq \alpha(q)} \quad \text { whenever } \quad c_{n}=1 .
\end{aligned}
$$

Lemma 2.3 implies that $\mathcal{V}_{q}$ is closed for any $q \in(1,2]$. Then using Lemma 2.2 we conclude that

$$
\mathcal{U}_{q} \subseteq \overline{\mathcal{U}_{q}} \subseteq \mathcal{V}_{q} \text { for every } q \in(1,2] .
$$

Moreover, the difference set $\mathcal{V}_{q} \backslash \mathcal{U}_{q}$ is at most countable. It might happen that $\mathcal{V}_{q}=\mathcal{U}_{q}$ (see Lemma 2.8 below).

For any $n \geq 1$ let $\mathcal{L}_{n}\left(\mathcal{U}_{q}^{\prime}\right)$ be the set of length $n$ subwords of sequences in $\mathcal{U}_{q}^{\prime}$, and let $\# A$ denote the cardinality of a set $A$. Then the topological entropy of $\mathcal{U}_{q}^{\prime}$ is defined by

$$
h_{\text {top }}\left(\mathcal{U}_{q}^{\prime}\right)=\lim _{n \rightarrow \infty} \frac{\log \# \mathcal{L}_{n}\left(\mathcal{U}_{q}^{\prime}\right)}{n}=\inf _{n \geq 1} \frac{\log \# \mathcal{L}_{n}\left(\mathcal{U}_{q}^{\prime}\right)}{n} .
$$

The above limit exists for each $q>1$ by [18, Lemma 2.1]. Moreover, the following lemma was proved in [18]:

Lemma 2.4. The Hausdorff dimension of $\mathcal{U}_{q}$ is given by

$$
\operatorname{dim}_{H} \mathcal{U}_{q}=\frac{h_{\text {top }}\left(\mathcal{U}_{q}^{\prime}\right)}{\log q}
$$

for every $q \in(1,2]$. Furthermore, the dimension function $D: q \mapsto \operatorname{dim}_{H} \mathcal{U}_{q}$ has a Devil's staircase behavior:

- $D$ is continuous, and has bounded variation in $(1,2]$;

- $D^{\prime}<0$ almost everywhere in $\left[q_{K L}, 2\right]$;

- $D(q)=0$ for all $1<q \leq q_{K L}$, and $D(q)>0$ for all $q>q_{K L}$.

We recall that $\mathcal{U}$ is the set of bases $q \in(1,2]$ in which 1 has a unique $q$-expansion, and $\mathcal{V}$ is the set of bases $q \in(1,2]$ in which 1 has a unique doubly infinite $q$-expansion.

The following lexicographical characterizations of $\mathcal{U}$, its closure $\overline{\mathcal{U}}$ and $\mathcal{V}$ are due to Komornik and Loreti [20] (see also, [9]).

\section{Lemma 2.5.}

(i) $q \in \mathcal{U} \backslash\{2\}$ if and only if $\alpha(q)=\left(\alpha_{i}(q)\right)$ satisfies

$$
\overline{\alpha(q)}<\alpha_{n+1}(q) \alpha_{n+2}(q) \cdots<\alpha(q) \text { for all } n \geq 1 .
$$

(ii) $q \in \overline{\mathcal{U}}$ if and only if $\alpha(q)=\left(\alpha_{i}(q)\right)$ satisfies

$$
\overline{\alpha(q)}<\alpha_{n+1}(q) \alpha_{n+2}(q) \cdots \leq \alpha(q) \text { for all } n \geq 1 .
$$

(iii) $q \in \mathcal{V}$ if and only if $\alpha(q)=\left(\alpha_{i}(q)\right)$ satisfies

$$
\overline{\alpha(q)} \leq \alpha_{n+1}(q) \alpha_{n+2}(q) \cdots \leq \alpha(q) \text { for all } n \geq 1 .
$$

Example 2.6. For the first two elements of $\mathcal{V}$ we have $\alpha(\varphi)=(10)^{\infty}$ and $\alpha\left(q_{f}\right)=(1100)^{\infty}$. 
By Lemma 2.5 it is clear that $\mathcal{U} \subset \overline{\mathcal{U}} \subset \mathcal{V}$. Furthermore, $\mathcal{V}$ is closed. In order to prove our main results we will also need the following topological properties of $\mathcal{U}, \overline{\mathcal{U}}$ and $\mathcal{V}$ (see [20]):

\section{Lemma 2.7.}

(i) $\overline{\mathcal{U}} \backslash \mathcal{U}$ is a countable dense subset of $\overline{\mathcal{U}}$, and $\mathcal{V} \backslash \overline{\mathcal{U}}$ is a discrete dense subset of $\mathcal{V}$.

(ii) For each $q \in \overline{\mathcal{U}} \backslash \mathcal{U}$ there exists a sequence $\left(p_{n}\right)$ in $\mathcal{U}$ that $p_{n} \nearrow q$ as $n \rightarrow \infty$.

(iii) For each $q \in \overline{\mathcal{U}} \backslash \mathcal{U}$ the quasi-greedy expansion $\alpha(q)$ is periodic.

(iv) For each $q \in \mathcal{V} \backslash(\overline{\mathcal{U}} \cup\{\varphi\})$ there exists a word $a_{1} \cdots a_{m}$ with $m \geq 2$ such that

$$
\alpha(q)=\left(a_{1} \cdots a_{m}^{+} \overline{a_{1} \cdots a_{m}^{+}}\right)^{\infty},
$$

where $\left(a_{1} \cdots a_{m}\right)^{\infty}$ satisfies (2.1).

Finally, we recall from [7] the following relation between the sets $\overline{\mathcal{U}}, \mathcal{V}, \mathcal{U}_{q}, \overline{\mathcal{U}_{q}}$ and $\mathcal{V}_{q}$.

\section{Lemma 2.8.}

(i) $\mathcal{U}_{q}$ is closed if and only if $q \in(1,2] \backslash \overline{\mathcal{U}}$.

(ii) $\mathcal{U}_{q}=\overline{\mathcal{U}_{q}}=\mathcal{V}_{q}$ if and only if $q \in(1,2] \backslash \mathcal{V}$.

(iii) Let $\left(r_{1}, r_{2}\right)$ be a connected component of $(1,2] \backslash \mathcal{V}$. Then $\mathcal{U}_{q}^{\prime}=\mathcal{U}_{r_{2}}^{\prime}$ for all $q \in\left(r_{1}, r_{2}\right]$.

\section{Description of $\mathcal{B}_{2}$}

In this section we describe the set $\mathcal{B}_{2}$ by using unique expansions. The following result is essentially equivalent to Theorem 1.1 (i):

Lemma 3.1. A number $q \in(1,2]$ belongs to $\mathcal{B}_{2}$ if and only if there exist two sequences $\left(c_{i}\right),\left(d_{i}\right) \in$ $\mathcal{U}_{q}^{\prime}$ satisfying the equality

$$
\left(1\left(c_{i}\right)\right)_{q}=\left(0\left(d_{i}\right)\right)_{q} .
$$

Proof. The sufficiency of the condition (3.1) is obvious. Conversely, assume that a real number $x$ has exactly two $q$-expansions, say $\left(a_{i}\right)$ and $\left(b_{i}\right)$. Then, assuming by symmetry that $\left(a_{i}\right)>$ $\left(b_{i}\right)$ lexicographically, there exists a first index $n$ such that $a_{n} \neq b_{n}$. Then $a_{n}=1, b_{n}=0$, $\left(a_{n+i}\right),\left(b_{n+i}\right) \in \mathcal{U}_{q}^{\prime}$, and (3.1) is satisfied with $\left(c_{i}\right)=\left(a_{n+i}\right)$ and $\left(d_{i}\right)=\left(b_{n+i}\right)$.

For each $q \in(1,2]$ we define

$$
A_{q}^{\prime}:=\left\{\left(c_{i}\right) \in \mathcal{U}_{q}^{\prime}: c_{1}=0\right\} .
$$

Each sequence $\left(c_{i}\right) \in A_{q}^{\prime}$ satisfies

$$
c_{n+1} c_{n+2} \cdots<\alpha(q) \text { for any } n \geq 0,
$$

and

$$
\mathcal{U}_{q}^{\prime}=\bigcup_{\mathbf{c} \in A_{q}^{\prime}}\{\mathbf{c}, \overline{\mathbf{c}}\}
$$

by Lemma 2.2

In the following improvement of Lemma 3.1 we use only sequences from $A_{q}^{\prime}$ : 
Lemma 3.2. A number $q \in(1,2]$ belongs to $\mathcal{B}_{2}$ if and only if $q$ is a zero of the function

$$
f_{\mathbf{c}, \mathbf{d}}(q):=(1 \mathbf{c})_{q}+(1 \mathbf{d})_{q}-\left(1^{\infty}\right)_{q}
$$

for some $\mathbf{c}, \mathbf{d} \in A_{q}^{\prime}$.

Proof. Let $q \in(1,2]$. Since $\mathcal{U}_{q}^{\prime}=\bigcup_{\mathbf{c} \in A_{q}^{\prime}}\{\mathbf{c}, \overline{\mathbf{c}}\}$, by Lemma 3.1 we have $q \in \mathcal{B}_{2}$ if and only if $q$ satisfies one of the following equations for some $\mathbf{c}, \mathbf{d} \in A_{q}^{\prime}$ :

$$
(1 \mathbf{c})_{q}=(0 \mathbf{d})_{q}, \quad(1 \mathbf{c})_{q}=(0 \overline{\mathbf{d}})_{q}, \quad(1 \overline{\mathbf{c}})_{q}=(0 \mathbf{d})_{q} \quad \text { and } \quad(1 \overline{\mathbf{c}})_{q}=(0 \overline{\mathbf{d}})_{q}
$$

It follows by reflection that the first and the forth equalities are equivalent. It remains to prove that the first and the third equalities never hold.

Since $\mathbf{d} \in A_{q}^{\prime}$, by Lemma 2.2 we have

$$
(0 \mathbf{d})_{q}<(00 \alpha(q))_{q}<\left(10^{\infty}\right)_{q} \leq(1 \mathbf{c})_{q} .
$$

Similarly, $(0 \mathbf{d})_{q}<(1 \overline{\mathbf{c}})_{q}$. This implies that $q \in \mathcal{B}_{2}$ if and only if $q$ is a zero of the function

$$
f_{\mathbf{c}, \mathbf{d}}(q)=(1 \mathbf{c})_{q}-(0 \overline{\mathbf{d}})_{q}=(1 \mathbf{c})_{q}+(1 \mathbf{d})_{q}-\left(1^{\infty}\right)_{q} .
$$

In view of Lemma 3.2 we are led to investigate the functions $f_{\mathbf{c}, \mathbf{d}}$ for $\mathbf{c}, \mathbf{d} \in A_{q}^{\prime}$.

Lemma 3.3. Let $q \in(1,2]$ and $\mathbf{c}, \mathbf{d} \in A_{q}^{\prime}$.

(i) The function $f_{\mathbf{c}, \mathbf{d}}$ is continuous and symmetric: $f_{\mathbf{c}, \mathbf{d}}=f_{\mathbf{d}, \mathbf{c}}$.

(ii) If $\tilde{\mathbf{c}} \in A_{q}^{\prime}$ and $\tilde{\mathbf{c}}>\mathbf{c}$, then $f_{\tilde{\mathbf{c}}, \mathbf{d}}>f_{\mathbf{c}, \mathbf{d}}$.

(iii) If $\tilde{\mathbf{d}} \in A_{q}^{\prime}$ and $\tilde{\mathbf{d}}>\mathbf{d}$, then $f_{\mathbf{c}, \tilde{\mathbf{d}}}>f_{\mathbf{c}, \mathbf{d}}$.

(iv) $f_{\mathbf{c}, \mathbf{d}}(2) \geq 0$.

Proof. (i) follows from the definition of $f_{\mathbf{c}, \mathbf{d}}$, (ii) follows from the definition of $f_{\mathbf{c}, \mathbf{d}}$ and the property of unique expansions that

$$
(1 \tilde{\mathbf{c}})_{q}>(1 \mathbf{c})_{q}
$$

for any $\tilde{\mathbf{c}}, \mathbf{c} \in A_{q}^{\prime}$ with $\tilde{\mathbf{c}}>\mathbf{c}$. (iii) follows from (i) and (ii). Finally, (iv) follows from (ii), (iii) and the equality

$$
f_{\mathbf{c}, \mathbf{d}}(2)=\left(10^{\infty}\right)_{2}+\left(10^{\infty}\right)_{2}-\left(1^{\infty}\right)_{2}=0
$$

when $\mathbf{c}=\mathbf{d}=0^{\infty}$ (the lexicographically smallest element of $A_{q}^{\prime}$ ).

Lemma 3.2 states that each $q \in \mathcal{B}_{2}$ is a zero of the function $f_{\mathbf{c}, \mathbf{d}}$ for some $\mathbf{c}, \mathbf{d} \in A_{q}^{\prime}$. We prove in our next lemma that no function $f_{\mathbf{c}, \mathbf{d}}$ with $\mathbf{c}, \mathbf{d} \in A_{q}^{\prime}$ can provide more than one element of $\mathcal{B}_{2} \cap[q, 2]$.

Since $\mathcal{B}_{2} \cap\left(1, q_{f}\right)$ contains only one element by Theorem 1.1 (iv): the positive zero $q_{s} \approx 1.71064$ of $q^{4}-2 q^{2}-q-1$, henceforth we restrict our attention to the set $\mathcal{B}_{2} \cap\left[q_{f}, 2\right]$.

Lemma 3.4. Let $q \in\left[q_{f}, 2\right]$ and $\mathbf{c}, \mathbf{d} \in A_{q}^{\prime}$.

(i) If $\mathbf{c} \geq 010^{\infty}$ or $\mathbf{d} \geq 010^{\infty}$, then $f_{\mathbf{c}, \mathbf{d}}(q)>0$.

(ii) If $\mathbf{c} \geq 0^{3} 10^{\infty}$ and $\mathbf{d} \geq 0^{2} 10^{\infty}$, or $\mathbf{c} \geq 0^{2} 10^{\infty}$ and $\mathbf{d} \geq 0^{3} 10^{\infty}$, then $f_{\mathbf{c}, \mathbf{d}}(q)>0$. 


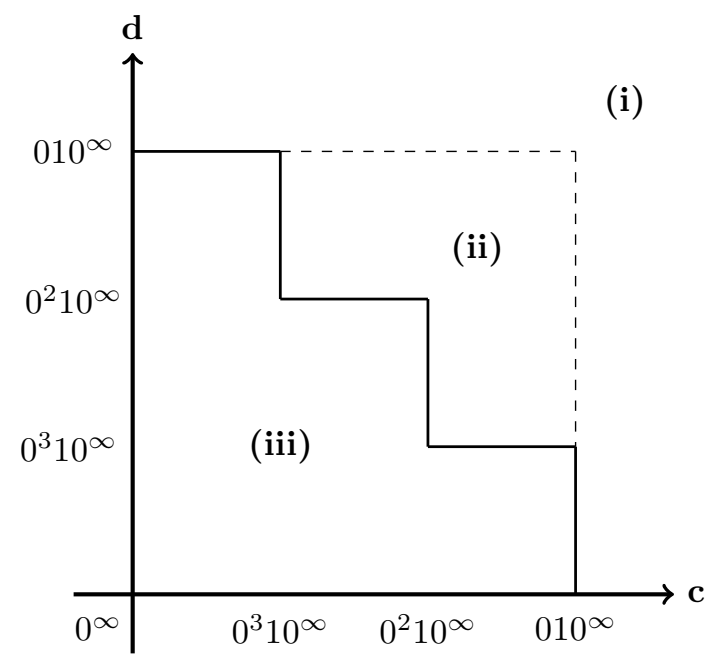

Figure 1: Different cases of Lemma 3.4

(iii) In all other cases the function $f_{\mathbf{c}, \mathbf{d}}$ is strictly increasing in $[q, 2]$.

Proof. (i) Let $q \in\left[q_{f}, 2\right]$. Applying Lemma 2.2 we see that $0^{\infty}$ is the lexicographically smallest element of $A_{q}^{\prime}$ and $010^{\infty} \notin A_{q}^{\prime}$. In view of Lemma 3.3 it suffices to prove that

$$
f_{\mathbf{c}, \mathbf{d}}(q) \geq 0 \text { when } \quad \mathbf{c}=010^{\infty} \text { and } \mathbf{d}=0^{\infty} .
$$

This follows from the following computation:

$$
\begin{aligned}
f_{\mathbf{c}, \mathbf{d}}(q) & =\left(1010^{\infty}\right)_{q}+\left(10^{\infty}\right)_{q}-\left(1^{\infty}\right) \\
& =\frac{2}{q}+\frac{1}{q^{3}}-\frac{1}{q-1} \\
& =\frac{q^{3}-2 q^{2}+q-1}{q^{3}(q-1)} \geq 0 .
\end{aligned}
$$

The last inequality holds because

$$
q^{3}-2 q^{2}+q-1 \geq q_{f}^{3}-2 q_{f}^{2}+q_{f}-1=0 .
$$

(ii) We recall that $\varphi_{3} \approx 1.83929$ is the Tribonacci number, the positive zero of $q^{3}-q^{2}-q-1$. We distinguish the following two cases: (a) $q \in\left[q_{f}, \varphi_{3}\right]$ and (b) $q \in\left[\varphi_{3}, 2\right]$.

Case (a): $q \in\left[q_{f}, \varphi_{3}\right]$. Since $\alpha\left(\varphi_{3}\right)=(110)^{\infty}$, by Lemma 2.1 we have $\alpha(q) \leq(110)^{\infty}$. Then by Lemma 2.2 it follows that if $\mathbf{c} \in A_{q}^{\prime}$ with $\mathbf{c} \geq 0^{k} 10^{\infty}$ for some $k \geq 0$, then $\mathbf{c} \geq 0^{k}(100)^{\infty}$. In view of Lemma 3.3 it suffices to prove

$$
f_{\mathbf{c}, \mathbf{d}}(q)>0 \text { when } \quad \mathbf{c}=0^{3}(100)^{\infty} \text { and } \mathbf{d}=0^{2}(100)^{\infty} .
$$

This follows from the following observation:

$$
\begin{aligned}
f_{\mathbf{c}, \mathbf{d}}(q) & =\left(10^{3}(100)^{\infty}\right)_{q}+\left(10^{2}(100)^{\infty}\right)_{q}-\left(1^{\infty}\right)_{q} \\
& =\frac{2}{q}+\frac{1}{q^{2}\left(q^{3}-1\right)}+\frac{1}{q\left(q^{3}-1\right)}-\frac{1}{q-1} \\
& =\frac{q^{4}-q^{3}-q^{2}-q+1}{q^{2}\left(q^{3}-1\right)}>0 .
\end{aligned}
$$


Here the last inequality follows by using the inequalities

$$
q^{4}-q^{3}-q^{2}-q+1 \geq q_{f}^{4}-q_{f}^{3}-q_{f}^{2}-q_{f}+1=2-q_{f}>0 .
$$

Case (b): $q \in\left[\varphi_{3}, 2\right]$. By Lemma 3.3 it suffices to prove that

$$
f_{\mathbf{c}, \mathbf{d}}(q)>0 \text { when } \quad \mathbf{c}=0^{3} 10^{\infty} \text { and } \mathbf{d}=0^{2} 10^{\infty} .
$$

This follows from the following computation:

$$
\begin{aligned}
f_{\mathbf{c}, \mathbf{d}}(q) & =\left(10^{3} 10^{\infty}\right)_{q}+\left(10^{2} 10^{\infty}\right)_{q}-\left(1^{\infty}\right)_{q} \\
& =\frac{2}{q}+\frac{1}{q^{4}}+\frac{1}{q^{5}}-\frac{1}{q-1}=\frac{q^{5}-2 q^{4}+q^{2}-1}{q^{5}(q-1)}>0 .
\end{aligned}
$$

The last inequality follows by using $q \geq \varphi_{3}$ and $\varphi_{3}^{3}=\varphi_{3}^{2}+\varphi_{3}+1$ :

$$
\begin{aligned}
q^{5}-2 q^{4}+q^{2}-1 & \geq \varphi_{3}^{5}-2 \varphi_{3}^{4}+\varphi_{3}^{2}-1 \\
& =-\varphi_{3}^{4}+\varphi_{3}^{3}+2 \varphi_{3}^{2}-1=\varphi_{3}^{2}-\varphi_{3}-1>0 .
\end{aligned}
$$

(iii) Let $q \in\left[q_{f}, 2\right]$ and $p_{1}, p_{2} \in[q, 2]$ with $p_{1}<p_{2}$. We will prove the inequality $f_{\mathbf{c}, \mathbf{d}}\left(p_{1}\right)<$ $f_{\mathbf{c}, \mathbf{d}}\left(p_{2}\right)$ by distinguishing two cases again (see Figure 1).

First case: $\mathbf{c}<0^{3} 10^{\infty}$ and $\mathbf{d}<010^{\infty}$, or $\mathbf{c}<010^{\infty}$ and $\mathbf{d}<0^{3} 10^{\infty}$. Observe that for any sequence $\left(a_{i}\right) \in\{0,1\}^{\infty}$ and for any positive integer $n$ the elementary inequality

$$
0 \leq\left(a_{1} a_{2} \cdots\right)_{p_{1}}-\left(a_{1} a_{2} \cdots\right)_{p_{2}} \leq\left(a_{1} \cdots a_{n} 1^{\infty}\right)_{p_{1}}-\left(a_{1} \cdots a_{n} 1^{\infty}\right)_{p_{2}}
$$

holds. By Lemma 3.3 it suffices to prove that

$$
f_{\mathbf{c}, \mathbf{d}}\left(p_{1}\right)<f_{\mathbf{c}, \mathbf{d}}\left(p_{2}\right) \quad \text { when } \quad \mathbf{c} \leq 0^{4} 1^{\infty} \text { and } \mathbf{d} \leq 0^{2} 1^{\infty} .
$$

Using (3.2) with $n=5$ and $n=3$ we get

$$
\begin{aligned}
f_{\mathbf{c}, \mathbf{d}}\left(p_{2}\right)-f_{\mathbf{c}, \mathbf{d}}\left(p_{1}\right)= & {\left[(1 \mathbf{c})_{p_{2}}-(1 \mathbf{c})_{p_{1}}\right]+\left[(1 \mathbf{d})_{p_{2}}-(1 \mathbf{d})_{p_{1}}\right]-\left[\left(1^{\infty}\right)_{p_{2}}-\left(1^{\infty}\right)_{p_{1}}\right] } \\
\geq & {\left[\left(10^{4} 1^{\infty}\right)_{p_{2}}-\left(10^{4} 1^{\infty}\right)_{p_{1}}\right]+\left[\left(10^{2} 1^{\infty}\right)_{p_{2}}-\left(10^{2} 1^{\infty}\right)_{p_{1}}\right] } \\
& -\left[\left(1^{\infty}\right)_{p_{2}}-\left(1^{\infty}\right)_{p_{1}}\right] \\
= & {\left[\left(10^{4} 1^{\infty}\right)_{p_{2}}+\left(10^{2} 1^{\infty}\right)_{p_{2}}-\left(1^{\infty}\right)_{p_{2}}\right]-\left[\left(10^{4} 1^{\infty}\right)_{p_{1}}+\left(10^{2} 1^{\infty}\right)_{p_{1}}-\left(1^{\infty}\right)_{p_{1}}\right] . }
\end{aligned}
$$

The required inequality $f_{\mathbf{c}, \mathbf{d}}\left(p_{2}\right)>f_{\mathbf{c}, \mathbf{d}}\left(p_{1}\right)$ follows by observing that the function

$$
g_{1}(x):=\left(10^{4} 1^{\infty}\right)_{x}+\left(10^{2} 1^{\infty}\right)_{x}-\left(1^{\infty}\right)_{x}=\frac{x^{5}-2 x^{4}+x^{2}+1}{x^{5}(x-1)}
$$

is strictly increasing in $\left[q_{f}, 2\right]$.

Second case: $\mathbf{c}<0^{2} 10^{\infty}$ and $\mathbf{d}<0^{2} 10^{\infty}$. Now using (3.2) with $n=4$ we get, similarly to the first case, the inequality

$$
f_{\mathbf{c}, \mathbf{d}}\left(p_{2}\right)-f_{\mathbf{c}, \mathbf{d}}\left(p_{1}\right) \geq\left[2\left(10^{3} 1^{\infty}\right)_{p_{2}}-\left(1^{\infty}\right)_{p_{2}}\right]-\left[2\left(10^{3} 1^{\infty}\right)_{p_{1}}-\left(1^{\infty}\right)_{p_{1}}\right] .
$$

Note that the function

$$
g_{2}(x):=2\left(10^{3} 1^{\infty}\right)_{x}-\left(1^{\infty}\right)_{x}=\frac{x^{4}-2 x^{3}+2}{x^{4}(x-1)}
$$

is strictly increasing in $\left[q_{f}, 2\right]$. Therefore we conclude that $f_{\mathbf{c}, \mathbf{d}}\left(p_{2}\right)>f_{\mathbf{c}, \mathbf{d}}\left(p_{1}\right)$. 
Let $q \in\left[q_{f}, 2\right]$ and set

$$
\Omega_{q}^{\prime}:=\left\{(\mathbf{c}, \mathbf{d}) \in A_{q}^{\prime} \times A_{q}^{\prime}: f_{\mathbf{c}, \mathbf{d}}(q) \leq 0\right\} .
$$

If $(\mathbf{c}, \mathbf{d}) \in \Omega_{q}^{\prime}$, then $f_{\mathbf{c}, \mathbf{d}}$ has a unique root in $[q, 2]$ by Lemmas 3.3 (iv) and 3.4 and this root, denoted by $q_{\mathbf{c}, \mathbf{d}}$, belongs to $\mathcal{B}_{2}$ by Lemma 3.2. We have

$$
\mathcal{B}_{2} \cap[q, 2]=\bigcup_{p \in[q, 2]}\left\{q_{\mathbf{c}, \mathbf{d}}:(\mathbf{c}, \mathbf{d}) \in \Omega_{p}^{\prime}\right\} .
$$

An element of $\mathcal{B}_{2} \cap\left[q_{f}, 2\right]$ may have multiple representations: see Remark 4.2 below.

Lemma 3.5. Let $q \in\left[q_{f}, 2\right]$.

(i) If $(\mathbf{c}, \mathbf{d}),(\tilde{\mathbf{c}}, \mathbf{d}) \in \Omega_{q}^{\prime}$ and $\tilde{\mathbf{c}}>\mathbf{c}$, then $q_{\tilde{\mathbf{c}}, \mathbf{d}}<q_{\mathbf{c}, \mathbf{d}}$;

(ii) If $(\mathbf{c}, \mathbf{d}),(\mathbf{c}, \tilde{\mathbf{d}}) \in \Omega_{q}^{\prime}$ and $\tilde{\mathbf{d}}>\mathbf{d}$, then $q_{\mathbf{c}, \tilde{\mathbf{d}}}<q_{\mathbf{c}, \mathbf{d}}$.

Proof. By symmetry we only prove (i).

Let $(\mathbf{c}, \mathbf{d}),(\tilde{\mathbf{c}}, \mathbf{d}) \in \Omega_{q}^{\prime}$ with $\tilde{\mathbf{c}}>\mathbf{c}$. Applying Lemma 3.3 and using the definitions of $q_{\mathbf{c}, \mathbf{d}}, q_{\tilde{\mathbf{c}}, \mathbf{d}}$ we have

$$
\begin{aligned}
f_{\tilde{\mathbf{c}}, \mathbf{d}}\left(q_{\tilde{\mathbf{c}}, \mathbf{d}}\right)=0=f_{\mathbf{c}, \mathbf{d}}\left(q_{\mathbf{c}, \mathbf{d}}\right) & =(1 \mathbf{c})_{q_{\mathbf{c}, \mathbf{d}}}+(1 \mathbf{d})_{q_{\mathbf{c}, \mathbf{d}}}-\left(1^{\infty}\right)_{q_{\mathbf{c}, \mathbf{d}}} \\
& <(1 \tilde{\mathbf{c}})_{q_{\mathbf{c}, \mathbf{d}}}+(1 \mathbf{d})_{q_{\mathbf{c}, \mathbf{d}}}-\left(1^{\infty}\right)_{q_{\mathbf{c}, \mathbf{d}}} \\
& =f_{\tilde{\mathbf{c}}, \mathbf{d}}\left(q_{\mathbf{c}, \mathbf{d}}\right) .
\end{aligned}
$$

Since $f_{\tilde{\mathbf{c}}, \mathbf{d}}$ is strictly increasing in $[q, 2]$ by Lemma 3.4 we conclude that $q_{\tilde{\mathbf{c}}, \mathbf{d}}<q_{\mathbf{c}, \mathbf{d}}$.

\section{Proof of Theorem 1.3, Theorem 1.5 (i)-(iv) and Theorem 1.7 (i)-(iii)}

We recall that $\mathcal{V}$ is the set of bases $q \in(1,2]$ in which 1 has a unique doubly infinite $q$-expansion; equivalently, $\mathcal{V}=\left\{q \in(1,2]: 1 \in \mathcal{V}_{q}\right\}$. In this section we show that $\mathcal{V} \backslash\{\varphi\} \subset \mathcal{B}_{2}$. Based on this observation we give new characterizations of $\mathcal{B}_{2}$ (Theorem 1.3). We also show that $\mathcal{B}_{2}$ is closed, $\mathcal{V} \backslash\{\varphi\} \subset \mathcal{B}_{2}^{(2)}$, and we conclude that the second smallest element $q_{f}$ of $\mathcal{B}_{2}$ is also the smallest accumulation point of $\mathcal{B}_{2}$ (Theorem 1.5).

We recall that $\mathcal{U}$ is the set of univoque bases $q \in(1,2]$ in which 1 has a unique $q$-expansion, i.e., $\mathcal{U}=\left\{q \in(1,2]: 1 \in \mathcal{U}_{q}\right\}$. First we show that its topological closure $\overline{\mathcal{U}}$ is a subset of $\mathcal{B}_{2}$ :

Lemma 4.1. We have $\overline{\mathcal{U}} \subset \mathcal{B}_{2}$, and even $\overline{\mathcal{U}} \subset \mathcal{B}_{2}^{(\infty)}$.

Proof. Since $\overline{\mathcal{U}}$ is a Cantor set and therefore $\overline{\mathcal{U}}^{(\infty)}=\overline{\mathcal{U}}$, it suffices to prove that $\overline{\mathcal{U}} \subset \mathcal{B}_{2}$. Furthermore, since $\mathcal{U} \subset \mathcal{B}_{2}$ by Theorem 1.1, it suffices to prove that $\overline{\mathcal{U}} \backslash \mathcal{U} \subset \mathcal{B}_{2}$.

Take $q \in \overline{\mathcal{U}} \backslash \mathcal{U}$ arbitrarily. By Lemma 2.7 (iii) there exists a word $a_{1} \cdots a_{m}$ such that

$$
\alpha(q)=\left(a_{1} \cdots a_{m}\right)^{\infty} .
$$

Suppose that $m$ is the smallest period of $\alpha(q)$. Then $m \geq 2$, and the greedy $q$-expansion of 1 is given by $\beta(q)=a_{1} \cdots a_{m}^{+} 0^{\infty}$. Observe that $\sigma^{n}(\beta(q))<\beta(q)$ for all $n \geq 1$ (cf. [25]). Then applying Lemma 2.5 (ii) it follows that

$$
\overline{a_{1} \cdots a_{m-i}} \leq a_{i+1} \cdots a_{m}<a_{i+1} \cdots a_{m}^{+} \leq a_{1} \cdots a_{m-i} \quad \text { for all } \quad 0<i<m .
$$


Since $q \in \overline{\mathcal{U}} \backslash \mathcal{U}$, by Lemmas 2.1 and 2.7 (ii) there exists a $p \in \mathcal{U} \cap(1, q)$ such that

$$
\alpha_{1}(p) \cdots \alpha_{m}(p)=\alpha_{1}(q) \cdots \alpha_{m}(q)=a_{1} \cdots a_{m} .
$$

In fact we could find infinitely many $p \in \mathcal{U} \cap(1, q)$ satisfying (4.2). Now we claim that

$$
\mathbf{c}:=\overline{a_{1} \cdots a_{m}^{+}} \alpha_{1}(p) \alpha_{2}(p) \cdots
$$

belongs to $A_{q}^{\prime}$, where $A_{q}^{\prime}$ is the set of sequences in $\mathcal{U}_{q}^{\prime}$ with a prefix 0 .

First we prove that $\mathbf{c} \in \mathcal{U}_{q}^{\prime}$. Since $p \in \mathcal{U} \cap(1, q)$, by Lemmas 2.1 and 2.5 (i) it follows that

$$
\overline{\alpha(q)}<\overline{\alpha(p)} \leq \sigma^{n}(\alpha(p)) \leq \alpha(p)<\alpha(q) \text { for any } n \geq 0 .
$$

Then by Lemma 2.2 it suffices to prove

$$
\overline{\alpha(q)}<\sigma^{i}(\mathbf{c})<\alpha(q) \text { for any } 0<i<m .
$$

By (4.1) it follows that for any $0<i<m$ we have

$$
\overline{a_{i+1} \cdots a_{m}^{+}}<\overline{a_{i+1} \cdots a_{m}} \leq a_{1} \cdots a_{m-i},
$$

which implies that $\sigma^{i}(\mathbf{c})<\alpha(q)$ for any $0<i<m$. Furthermore, by (4.1) we have

$$
\overline{a_{i+1} \cdots a_{m}^{+}} \geq \overline{a_{1} \cdots a_{m-i}} \quad \text { and } \quad a_{1} \cdots a_{i} \geq \overline{a_{m-i+1} \cdots a_{m}} .
$$

This, together with (4.2), implies that

$$
\begin{aligned}
\sigma^{i}(\mathbf{c}) & =\overline{a_{i+1} \cdots a_{m}^{+}} \alpha_{1}(p) \alpha_{2}(p) \cdots \\
& =\overline{a_{i+1} \cdots a_{m}^{+}} a_{1} \cdots a_{i} \alpha_{i+1}(p) \alpha_{i+2}(p) \cdots \\
& \geq \overline{a_{1} \cdots a_{m}} \overline{\alpha(p)} \\
& >\overline{a_{1} \cdots a_{m}} \overline{\alpha(q)}=\overline{\alpha(q)} ;
\end{aligned}
$$

the last equality holds because $\alpha(q)=\left(a_{1} \cdots a_{m}\right)^{\infty}$. Therefore, $\mathbf{c} \in \mathcal{U}_{q}^{\prime}$. Since $\overline{a_{1}}=0$, we conclude that $\mathbf{c} \in A_{q}^{\prime}$.

Now take $\mathbf{d}:=0^{m} \overline{\alpha(p)}$. Then by the above reasoning we also have $\mathbf{d} \in A_{q}^{\prime}$. In view of Lemma 3.2 the following calculation shows that $q \in \mathcal{B}_{2}$ :

$$
\begin{aligned}
f_{\mathbf{c}, \mathbf{d}}(q) & =(1 \mathbf{c})_{q}+(1 \mathbf{d})_{q}-\left(1^{\infty}\right)_{q} \\
& =\left(1 \overline{a_{1} \cdots a_{m}^{+}} \alpha(p)\right)_{q}+\left(10^{m} \overline{\alpha(p)}\right)_{q}-\left(1^{\infty}\right)_{q} \\
& =\left(2 \overline{a_{1} \cdots a_{m}^{+}} 1^{\infty}\right)_{q}-\left(1^{\infty}\right)_{q} \\
& =\left(10^{\infty}\right)_{q}-\left(0 a_{1} \cdots a_{m}^{+} 0^{\infty}\right)_{q}=0 ;
\end{aligned}
$$

the last equality holds because $\beta(q)=a_{1} \cdots a_{m}^{+} 0^{\infty}$.

Remark 4.2. The proof of Lemma 4.1 shows that each $q \in \overline{\mathcal{U}} \backslash \mathcal{U} \subset \mathcal{B}_{2}$ has infinitely many representations, i.e., $q=q_{\mathbf{c}, \mathbf{d}}$ for infinitely many pairs $(\mathbf{c}, \mathbf{d}) \in \Omega_{q}^{\prime}$.

The preceding lemma may be improved:

Lemma 4.3. $\mathcal{V} \backslash\{\varphi\} \subset \mathcal{B}_{2}$. 
Proof. By Lemma 4.1 it remains to prove that each $q \in \mathcal{V} \backslash(\overline{\mathcal{U}} \cup\{\varphi\})$ belongs to $\mathcal{B}_{2}$. Set

$$
\mathcal{V}^{\prime}=\left\{\left(a_{i}\right) \in\{0,1\}^{\infty}: \overline{\left(a_{i}\right)} \leq\left(a_{n+i}\right) \leq\left(a_{i}\right) \text { for all } n \geq 1\right\}
$$

By Lemmas 2.5 (iii) and 2.7 (iv) there exists a block $a_{1} \cdots a_{m}$ with $m \geq 2$ such that

$$
\alpha(q)=\left(a_{1} \cdots a_{m}^{+} \overline{a_{1} \cdots a_{m}^{+}}\right)^{\infty} \in \mathcal{V}^{\prime} \quad \text { and } \quad\left(a_{1} \cdots a_{m}\right)^{\infty} \in \mathcal{V}^{\prime} .
$$

We claim that

$$
\mathbf{c}=\overline{a_{1} \cdots a_{m}^{+}}\left(a_{1} \cdots a_{m}\right)^{\infty}
$$

belongs to $A_{q}^{\prime}$.

Since both $\alpha(q)=\left(a_{1} \cdots a_{m}^{+} \overline{a_{1} \cdots a_{m}^{+}}\right)^{\infty}$ and $\left(a_{1} \cdots a_{m}\right)^{\infty}$ belong to $\mathcal{V}^{\prime}$, we have

$$
\overline{a_{i+1} \cdots a_{m}^{+}}<\overline{a_{i+1} \cdots a_{m}} \leq a_{1} \cdots a_{m-i}
$$

and

$$
\overline{a_{i+1} \cdots a_{m}^{+}} a_{1} \cdots a_{i} \geq \overline{a_{1} \cdots a_{m-i}} \overline{a_{m-i+1} \cdots a_{m}}>\overline{a_{1} \cdots a_{m}^{+}}
$$

for all $0<i<m$. This implies the relations

$$
\left.\overline{\left(a_{1} \cdots a_{m}^{+}\right.} a_{1} \cdots a_{m}^{+}\right)^{\infty}<\sigma^{i}(\mathbf{c})<\left(a_{1} \cdots a_{m}^{+} \overline{a_{1} \cdots a_{m}^{+}}\right)^{\infty}
$$

for all $i>0$. Hence $\mathbf{c} \in \mathcal{U}_{q}^{\prime}$. Since $\overline{a_{1}}=0$, we conclude that $\mathbf{c} \in A_{q}^{\prime}$.

Let $\mathbf{d}:=0^{2 m}\left(\overline{a_{1} \cdots a_{m}}\right)^{\infty}$, then $\mathbf{d} \in A_{q}^{\prime}$. We complete the proof of $q \in \mathcal{B}_{2}$ by showing that $f_{\mathbf{c}, \mathbf{d}}(q)=0$. This follows from the following computation:

$$
\begin{aligned}
f_{\mathbf{c}, \mathbf{d}}(q) & =\left(\overline{\left(a_{1} \cdots a_{m}^{+}\right.}\left(a_{1} \cdots a_{m}\right)^{\infty}\right)_{q}+\left(10^{2 m}\left(\overline{a_{1} \cdots a_{m}}\right)^{\infty}\right)_{q}-\left(1^{\infty}\right)_{q} \\
& =\left(2 \overline{a_{1} \cdots a_{m}^{+}} a_{1} \cdots a_{m} 1^{\infty}\right)_{q}-\left(1^{\infty}\right)_{q} \\
& =\left(10^{\infty}\right)_{q}-\left(0 a_{1} \cdots a_{m}^{+} \overline{a_{1} \cdots a_{m}} 0^{\infty}\right)_{q}=0 ;
\end{aligned}
$$

the last equality holds because $\beta(q)=a_{1} \cdots a_{m}^{+} \overline{a_{1} \cdots a_{m}} 0^{\infty}$.

We recall that $\mathcal{U}_{q}$ is the set of $x \in I_{q}$ having a unique $q$-expansion, $\overline{\mathcal{U}_{q}}$ is its topological closure, and $\mathcal{V}_{q}$ is the set of $x \in I_{q}$ having at most one doubly infinite $q$-expansion. We also recall that $\mathcal{U}_{q} \subseteq \overline{\mathcal{U}_{q}} \subseteq \mathcal{V}_{q}$ for all $q \in(1,2]$.

Lemma 4.3 allows us to give new characterizations of $\mathcal{B}_{2}$ :

Proposition 4.4. Set

$$
\mathcal{C}_{2}:=\left\{q \in(1,2]: 1 \in \overline{\mathcal{U}_{q}}-\overline{\mathcal{U}_{q}}\right\} \quad \text { and } \quad \mathcal{D}_{2}:=\left\{q \in(1,2]: 1 \in \mathcal{V}_{q}-\mathcal{V}_{q}\right\} .
$$

Then $\mathcal{B}_{2}=\mathcal{C}_{2}=\mathcal{D}_{2} \backslash\{\varphi\}$.

Proof. We already know from Theorem 1.1 (i) that

$$
\mathcal{B}_{2}=\left\{q \in(1,2]: 1 \in \mathcal{U}_{q}-\mathcal{U}_{q}\right\} .
$$

Since $\mathcal{U}_{q} \subseteq \overline{\mathcal{U}_{q}} \subseteq \mathcal{V}_{q}$ for all $q \in(1,2]$, we have

$$
\mathcal{B}_{2} \subseteq \mathcal{C}_{2} \subseteq \mathcal{D}_{2}
$$


Since $\overline{\mathcal{U}_{q}}=\mathcal{U}_{q}$ for all $q \in(1,2] \backslash \overline{\mathcal{U}}$ by Lemma 2.8 (i), we infer from Lemma 4.1 that

$$
\mathcal{C}_{2} \subseteq \mathcal{B}_{2} \cup \overline{\mathcal{U}}=\mathcal{B}_{2}
$$

Combining this with (4.4) we conclude that $\mathcal{C}_{2}=\mathcal{B}_{2}$.

Next, since $\mathcal{V}_{q}=\mathcal{U}_{q}$ for all $q \in(1,2] \backslash \mathcal{V}$ by Lemma 2.8 (ii), using also Lemma 4.3 we obtain that

$$
\mathcal{D}_{2} \subseteq \mathcal{B}_{2} \cup \mathcal{V}=\mathcal{B}_{2} \cup\{\varphi\}
$$

Observe that the condition $1 \in \mathcal{V}_{q}-\mathcal{V}_{q}$ is satisfied for $q=\varphi$ because $0,1 \in \mathcal{V}_{\varphi}$. This implies that $\varphi \in \mathcal{D}_{2}$. Combining this with (4.4) and $\varphi \notin \mathcal{B}_{2}$ we conclude that $\mathcal{B}_{2}=\mathcal{D}_{2} \backslash\{\varphi\}$.

Lemma 4.5. $\mathcal{B}_{2}$ is compact.

Proof. Since $\mathcal{B}_{2}$ is bounded, it suffices to prove that its complement is open. Since by Theorem 1.1 (iv) that $\mathcal{B}_{2}$ has a smallest point $q_{s} \approx 1.71064$, it suffices to show that $\left(q_{s}, 2\right] \backslash \mathcal{B}_{2}$ is a neighborhood of each of its points.

Fix $q \in\left(q_{s}, 2\right] \backslash \mathcal{B}_{2}$ arbitrarily. Since $\varphi \approx 1.61803<q_{s}$, we have $q \notin \mathcal{V}$ by Lemma 4.3. Consider the connected component $\left(r_{1}, r_{2}\right)$ of the open set $(1,2) \backslash \mathcal{V}$ that contains $q$.

Since $q \notin \overline{\mathcal{U}}$, by Lemma 2.8 (i) the set $\mathcal{U}_{q}$ is compact, and hence $\mathcal{U}_{q}-\mathcal{U}_{q}$ is compact. Since by Theorem 1.1 (i) that $q \notin \mathcal{B}_{2}$ is equivalent to $1 \notin \mathcal{U}_{q}-\mathcal{U}_{q}$, hence $\operatorname{dist}\left(1, \mathcal{U}_{q}-\mathcal{U}_{q}\right)>0$.

By Lemma 2.8 (iii) we have $\mathcal{U}_{p}^{\prime}=\mathcal{U}_{q}^{\prime}$ for all $p \in\left(r_{1}, r_{2}\right)$. Therefore the sets $\mathcal{U}_{p}$ depend continuously on $p \in\left(r_{1}, r_{2}\right)$, and hence the inequality $\operatorname{dist}\left(1, \mathcal{U}_{p}-\mathcal{U}_{p}\right)>0$ remains valid in a small neighborhood $G$ of $q$. Then $1 \notin \mathcal{U}_{p}-\mathcal{U}_{p}$, i.e., $p \notin \mathcal{B}_{2}$ for all $p \in G$.

Next we improve Lemma 4.3 each $q \in \mathcal{V} \backslash\{\varphi\}$ is even an accumulation point of $\mathcal{B}_{2}$. In fact, the following stronger result holds:

Lemma 4.6. $\mathcal{V} \backslash\{\varphi\} \subset \mathcal{B}_{2}^{(2)}$.

Proof. In view of Lemma 4.1 it suffices to show that each $q \in \mathcal{V} \backslash(\overline{\mathcal{U}} \cup\{\varphi\})$ belongs to $\mathcal{B}_{2}^{(2)}$.

Take $q \in \mathcal{V} \backslash(\overline{\mathcal{U}} \cup\{\varphi\})$ arbitrarily. By Lemma 2.7 there exists a word $a_{1} \cdots a_{m}$ with $m \geq 2$ such that

$$
\alpha(q)=\left(a_{1} \cdots a_{m}^{+} \overline{a_{1} \cdots a_{m}^{+}}\right)^{\infty} \in \mathcal{V}^{\prime} \quad \text { and } \quad\left(a_{1} \cdots a_{m}\right)^{\infty} \in \mathcal{V}^{\prime},
$$

where $\mathcal{V}^{\prime}$ is defined in (4.3). The proof of Lemma 4.3 shows that $q=q_{\mathbf{c}, \mathbf{d}}$, where

$$
\mathbf{c}=\overline{a_{1} \cdots a_{m}^{+}}\left(a_{1} \cdots a_{m}\right)^{\infty} \in A_{q}^{\prime} \quad \text { and } \quad \mathbf{d}=0^{2 m}\left(\overline{a_{1} \cdots a_{m}}\right)^{\infty} \in A_{q}^{\prime} .
$$

Set

$$
\mathbf{d}_{k}=0^{2 m}\left(\overline{a_{1} \cdots a_{m}}\right)^{k}\left(\overline{a_{1} \cdots a_{m}^{+}} a_{1} \cdots a_{m}^{+}\right)^{\infty}, \quad k=1,2, \ldots
$$

We claim that $\mathbf{d}_{k} \in A_{p}^{\prime}$ for all $k \geq 1$ and $p \in(q, 2]$.

It suffices to prove that

$$
\overline{\alpha(p)}<\sigma^{i}\left(\left(\overline{a_{1} \cdots a_{m}}\right)^{k}\left(\overline{a_{1} \cdots a_{m}^{+}} a_{1} \cdots a_{m}^{+}\right)^{\infty}\right)<\alpha(p) \text { for all } i \geq 0 .
$$

Since $\left(a_{1} \cdots a_{m}^{+} \overline{a_{1} \cdots a_{m}^{+}}\right)^{\infty}=\alpha(q) \in \mathcal{V}^{\prime}$ and $\alpha(p)>\alpha(q)$, we infer from Lemmas 2.1 and 2.5 that

$$
\overline{\alpha(p)}<\overline{\alpha(q)} \leq \sigma^{i}\left(\left(\overline{a_{1} \cdots a_{m}^{+}} a_{1} \cdots a_{m}^{+}\right)^{\infty}\right) \leq \alpha(q)<\alpha(p) \text { for all } i \geq 0 .
$$


It remains to prove (4.5) for $0 \leq i<m k$. Since $\left(a_{1} \cdots a_{m}\right)^{\infty} \in \mathcal{V}^{\prime}$ we have

$$
\overline{a_{1} \cdots a_{m}^{+}}<\overline{a_{i+1} \cdots a_{m} a_{1} \cdots a_{i}}<a_{1} \cdots a_{m}^{+} \text {for all } 0 \leq i<m,
$$

and this implies (4.5).

We have shown that $\mathbf{d}_{k} \in A_{p}^{\prime}$ for all $p>q$ and $k \geq 1$. Since $\mathbf{d}_{k}<\mathbf{d}$, using the equality $\alpha(q)=\left(a_{1} \cdots a_{m}^{+} \overline{a_{1} \cdots a_{m}^{+}}\right)^{\infty}$ it follows that

$$
\begin{aligned}
f_{\mathbf{c}, \mathbf{d}_{k}}(q) & =(1 \mathbf{c})_{q}+\left(1 \mathbf{d}_{k}\right)_{q}-\left(1^{\infty}\right)_{q} \\
& =(1 \mathbf{c})_{q}+\left(10^{2 m}\left(\overline{a_{1} \cdots a_{m}}\right)^{k+1} 0^{\infty}\right)_{q}-\left(1^{\infty}\right)_{q} \\
& <(1 \mathbf{c})_{q}+(1 \mathbf{d})_{q}-\left(1^{\infty}\right)_{q}=f_{\mathbf{c}, \mathbf{d}}(q)=0 .
\end{aligned}
$$

By Lemma 3.4 this implies that $f_{\mathbf{c}, \mathbf{d}_{k}}$ has a unique root $q_{\mathbf{c}, \mathbf{d}_{k}}$ in $(q, 2]$, and then it belongs to $\mathcal{B}_{2}$. Since $\mathbf{d}_{k}$ strictly increases to $\mathbf{d}$ as $k \rightarrow \infty$, we conclude by Lemma 3.5 and continuity that $q_{\mathbf{c}, \mathbf{d}_{k}} \searrow q$ as $k \rightarrow \infty$.

By the same argument as above we can also show that for each fixed $k \geq 1$,

$$
q_{\mathbf{c}_{\ell}, \mathbf{d}_{k}} \in \mathcal{B}_{2}, \quad \text { and } \quad q_{\mathbf{c}_{\ell}, \mathbf{d}_{k}} \nearrow q_{\mathbf{c}, \mathbf{d}_{k}} \quad \text { as } \quad \ell \rightarrow \infty,
$$

where

$$
\mathbf{c}_{\ell}:=\overline{a_{1} \cdots a_{m}^{+}}\left(a_{1} \cdots a_{m}\right)^{\ell}\left(a_{1} \cdots a_{m}^{+} \overline{a_{1} \cdots a_{m}^{+}}\right)^{\infty} .
$$

Therefore $q \in \mathcal{B}_{2}^{(2)}$.

Now we apply the above results to prove some theorems stated in the introduction:

Proof of Theorem 1.3. We combine Proposition 4.4 and Theorem 1.1 (i).

Proof of Theorem 1.5 (I)-(IV). (i)-(iii) were established in Lemmas 4.5, 4.3 and 4.6, respectively.

(iv) We know that the smallest element $q_{s}$ of $\mathcal{B}_{2}$ is isolated. The second smallest element $q_{f}$ of $\mathcal{B}_{2}$ belongs to $\mathcal{V}$ (it is the smallest element in $\mathcal{V}$ after $\varphi$ ), hence it is the smallest accumulation point of $\mathcal{B}_{2}$ by the preceding lemma. Indeed, $q_{f} \approx 1.75488$ satisfies $q_{f}^{4}=q_{f}^{3}+q_{f}^{2}+1$. Hence $\alpha\left(q_{f}\right)=(1100)^{\infty}$, so that $q_{f} \in \mathcal{V} \backslash\{\varphi\}$ by Lemma 2.5

Theorem 1.5(v)-(vi) will be proved in Section 7 below.

Proof of Theorem 1.7 (I)-(III). (i) and (ii) coincide with Lemmas 4.1 and 4.6, respectively. (iii) follows from Lemma 4.6 and Theorem 1.5 (iv).

Theorem 1.7 (iv)-(vi) will be proved in Sections 6 and 7 below.

\section{Explicit description of unique expansions}

In this section we give the detailed description of unique expansions. This will be used to investigate the derived sets $\mathcal{B}_{2}^{(j)}$ and $\mathcal{B}_{2}^{(\infty)}$ in the next sections.

We recall that $\overline{\mathcal{U}}$ is a Cantor set, and its smallest element is the Komornik-Loreti constant $q_{K L}$. Furthermore, its complement is the union of countably many disjoint open intervals:

$$
(1,2] \backslash \overline{\mathcal{U}}=(1,2) \backslash \overline{\mathcal{U}}=\bigcup\left(q_{0}, q_{0}^{*}\right),
$$


where $q_{0}$ runs over $\{1\} \cup(\overline{\mathcal{U}} \backslash \mathcal{U})$ and $q_{0}^{*}$ runs over a proper subset $\mathcal{U}^{*}$ of $\mathcal{U}$, formed by the $d e$ Vries-Komornik numbers. The first connected component is $\left(1, q_{K L}\right)$. Each left endpoint $q_{0}$ is an algebraic integer, and each right endpoint $q_{0}^{*}$ is a transcendental number.

Since $q_{0} \in\{1\} \cup(\overline{\mathcal{U}} \backslash \mathcal{U})$, we have $\alpha\left(q_{0}\right)=\left(a_{1} \cdots a_{m}\right)^{\infty}$ with a smallest periodic block $a_{1} \cdots a_{m}$ satisfying

$$
\overline{a_{i} \cdots a_{m} a_{1} \cdots a_{i-1}} \leq a_{1} \cdots a_{m}^{+} \quad \text { and } \quad a_{i} \cdots a_{m}^{+} \overline{a_{1} \cdots a_{i-1}} \leq a_{1} \cdots a_{m}^{+}
$$

for all $0<i \leq m$. This implies that $\beta\left(q_{0}\right)=a_{1} \cdots a_{m}^{+} 0^{\infty}$. Here we use the convention $\alpha(1):=0^{\infty}$ for $q_{0}=1$, although $0^{\infty}$ is not a 1-expansion of 1 . However $\beta(1)=10^{\infty}$ is a 1-expansion of 1 in the natural sense. (We have not defined $q$-expansions for $q=1$.)

We will say that the interval $\left(q_{0}, q_{0}^{*}\right)$ is the connected component of $(1,2] \backslash \overline{\mathcal{U}}$ generated by $a_{1} \cdots a_{m}$. In particular, the interval $\left(1, q_{K L}\right)$ is called the connected component of $(1,2] \backslash \overline{\mathcal{U}}$ generated by the word $a_{1}=0$.

We recall that $\mathcal{V}$ is the set of bases $q \in(1,2]$ such that 1 has a unique doubly infinite $q$ expansion, and that $\overline{\mathcal{U}} \subset \mathcal{V}$. Furthermore, for each connected component $\left(q_{0}, q_{0}^{*}\right)$ of $(1,2] \backslash \overline{\mathcal{U}}$, we have

$$
\mathcal{V} \cap\left(q_{0}, q_{0}^{*}\right)=\left\{q_{n}: n=1,2, \ldots\right\},
$$

where $q_{0}<q_{1}<q_{2}<\cdots<q_{0}^{*}$ and $q_{n} \nearrow q_{0}^{*}$ as $n \rightarrow \infty$. Therefore

$$
\left(q_{0}, q_{0}^{*}\right) \backslash \mathcal{V}=\bigcup_{n=0}^{\infty}\left(q_{n}, q_{n+1}\right)
$$

Let $\left(q_{0}, q_{0}^{*}\right)$ be the connected component of $(1,2] \backslash \overline{\mathcal{U}}$ generated by $\omega_{0}^{-}=a_{1} \cdots a_{m}$. We define a sequence of words recursively by the formulas $\omega_{0}:=a_{1} \cdots a_{m}^{+}$and

$$
\omega_{n+1}:=\omega_{n}{\overline{\omega_{n}}}^{+}, \quad n=0,1, \ldots
$$

For example,

$$
\omega_{1}=a_{1} \cdots a_{m}^{+} \overline{a_{1} \cdots a_{m}}, \quad \text { and } \quad \omega_{2}=a_{1} \cdots a_{m}^{+} \overline{a_{1} \cdots a_{m}} \overline{a_{1} \cdots a_{m}^{+}} a_{1} \cdots a_{m}^{+} .
$$

Observe that $\omega_{n}$ is a word of length $2^{n} m$.

We recall from [20, 7, 9] the relations

$$
\alpha\left(q_{n}\right)=\left(\omega_{n}^{-}\right)^{\infty}=\left(\omega_{n-1} \overline{\omega_{n-1}}\right)^{\infty}, \quad \text { and } \quad \beta\left(q_{n}\right)=\omega_{n} 0^{\infty}
$$

for all $n=0,1, \ldots$ It follows that the infinite sequence $\alpha\left(q_{0}^{*}\right)=\lim _{n \rightarrow \infty} \alpha\left(q_{n}\right)$ begins with

$$
\omega_{0} \bar{\omega}_{0}+\overline{\omega_{0}} \omega_{0} \overline{\omega_{0}} \omega_{0}^{-} \omega_{0}{\overline{\omega_{0}}}^{+} .
$$

The construction shows that for $q_{0}^{*}=q_{K L}$ the quasi-greedy expansion $\alpha\left(q_{0}^{*}\right)$ is the truncated Thue-Morse sequence (see Section 7 ).

The main purpose of this section is to describe explicitly the difference sets $\mathcal{U}_{q}^{\prime} \backslash \mathcal{U}_{q_{0}}^{\prime}$ for all $q \in\left(q_{0}, q_{0}^{*}\right]$ for any connected component $\left(q_{0}, q_{0}^{*}\right)$ of $(1,2] \backslash \overline{\mathcal{U}}$.

We need the following result from [21]:

Lemma 5.1. Let $\left(q_{0}, q_{0}^{*}\right)$ be a connected component of $(1,2] \backslash \overline{\mathcal{U}}$ generated by $\omega_{0}^{-}$, and let $\left(c_{i}\right) \in \mathcal{U}_{q_{0}^{*}}^{\prime}$.

- If $c_{\ell}=0$ for some $\ell \geq 1$ and $c_{\ell+1} \cdots c_{\ell+2^{k} m}=\omega_{k}$ for some $k \geq 0$, then the next block of length $2^{k} m$ is

$$
c_{\ell+2^{k} m+1} \cdots c_{\ell+2^{k+1} m}=\overline{\omega_{k}} \quad \text { or } \quad c_{\ell+2^{k} m+1} \cdots c_{\ell+2^{k+1} m}={\overline{\omega_{k}}}^{+} .
$$


- Symmetrically, if $c_{\ell}=1$ for some $\ell \geq 1$ and $c_{\ell+1} \cdots c_{\ell+2^{k} m}=\overline{\omega_{k}}$ for some $k \geq 0$, then the next block of length $2^{k} m$ is

$$
c_{\ell+2^{k} m+1} \cdots c_{\ell+2^{k+1} m}=\omega_{k} \quad \text { or } \quad c_{\ell+2^{k} m+1} \cdots c_{\ell+2^{k+1} m}=\omega_{k}{ }^{-} .
$$

We also need [21, Lemma 4.2]:

Lemma 5.2. Let $\left(q_{0}, q_{0}^{*}\right)$ be a connected component of $(1,2] \backslash \overline{\mathcal{U}}$ generated by $\omega_{0}^{-}$. Then for any $n \geq 0$ the word $\omega_{n}=\theta_{1} \cdots \theta_{2^{n} m}$ satisfies

$$
\overline{\theta_{1} \cdots \theta_{2^{n} m-i}}<\theta_{i+1} \cdots \theta_{2^{n} m} \leq \theta_{1} \cdots \theta_{2^{n} m-i}
$$

for all $0 \leq i<2^{n} m$.

Now we prove the main result of this section:

Theorem 5.3. Let $\left(q_{0}, q_{0}^{*}\right)$ be a connected component of $(1,2] \backslash \overline{\mathcal{U}}$ generated by $\omega_{0}^{-}$. Then $\mathcal{U}_{q_{0}^{*}}^{\prime} \backslash \mathcal{U}_{q_{0}}^{\prime}$ is formed by the sequences of the form

$$
\omega\left(\omega_{0}^{-}\right)^{j_{0}}\left(\omega_{k_{1}} \overline{\omega_{k_{1}}}\right)^{j_{1}}\left(\omega_{k_{1}} \overline{\omega_{k_{2}}}\right)^{s_{2}}\left(\omega_{k_{2}} \overline{\omega_{k_{2}}}\right)^{j_{2}}\left(\omega_{k_{2}} \overline{\omega_{k_{3}}}\right)^{s_{3}} \ldots
$$

and their reflections, where the word $\omega$ satisfies $\omega\left(\omega_{k}^{-}\right)^{\infty} \in \mathcal{U}_{q_{0}^{*}}^{\prime}$ for all $k \geq 0$, and the indices $k_{r}$, $j_{r}, s_{r}$ satisfy the following conditions:

$$
\begin{aligned}
& 0 \leq k_{1}<k_{2}<\cdots \quad \text { are integers; } \\
& j_{r} \in\{0,1, \cdots\} \text { or } j_{r}=\infty \text { for each } r ; \\
& s_{r} \in\{0,1\} \quad \text { for all } r ; \\
& j_{r}=\infty \Longrightarrow s_{u}=j_{u}=0 \text { for all } u>r .
\end{aligned}
$$

Remark 5.4. We may assume in Theorem 5.3 that $s_{r}+j_{r}+s_{r+1} \geq 1$ whenever $r \geq 2$ and $j_{r}<\infty$. Indeed, in case $s_{r}+j_{r}+s_{r+1}=0$ the word $\omega_{k_{r}}$ is missing, and we may renumber the blocks.

PROOF. First we show that each sequence in $\mathcal{U}_{q_{0}^{*}}^{\prime} \backslash \mathcal{U}_{q_{0}}^{\prime}$ or its reflection is of the form (5.4).

Fix $\left(c_{i}\right) \in \mathcal{U}_{q_{0}^{*}}^{\prime} \backslash \mathcal{U}_{q_{0}}^{\prime}$ arbitrarily. There exists a smallest integer $N \geq 1$ such that

$$
c_{N}=0 \quad \text { and } \quad c_{N+1} c_{N+2} \cdots \geq \alpha\left(q_{0}\right)=\left(\omega_{0}^{-}\right)^{\infty}
$$

or

$$
c_{N}=1, \quad c_{N+1} c_{N+2} \cdots \leq \overline{\alpha\left(q_{0}\right)}=\left({\overline{\omega_{0}}}^{+}\right)^{\infty} .
$$

Since the reflection $\overline{\left(c_{i}\right)}$ of $\left(c_{i}\right)$ also belongs to $\mathcal{U}_{q_{0}^{*}}^{\prime}$, we may assume that (5.5) holds.

Then setting $\omega_{0}^{-}=: a_{1} \ldots a_{m}$ we have

$$
c_{N+1} \cdots c_{N+m-1}=a_{1} \cdots a_{m-1},
$$

and for $0<n<N$ we have

$$
\begin{aligned}
& c_{n+1} \cdots c_{n+m} \leq a_{1} \ldots a_{m} \quad \text { if } \quad c_{n}=0 \\
& c_{n+1} \cdots c_{n+m} \geq \overline{a_{1} \cdots a_{m}} \quad \text { if } \quad c_{n}=1 .
\end{aligned}
$$

Indeed, (5.6) follows from the relations

$$
\alpha\left(q_{0}\right) \leq c_{N+1} c_{N+2} \cdots<\alpha\left(q_{0}^{*}\right),
$$


because both $\alpha\left(q_{0}\right)$ and $\alpha\left(q_{0}^{*}\right)$ start with $a_{1} \cdots a_{m-1}$, while (5.7) follows from the minimality of $N$ implying

$$
\begin{array}{lll}
c_{n+1} c_{n+2} \cdots<\alpha\left(q_{0}\right) & \text { if } & c_{n}=0, \\
c_{n+1} c_{n+2} \cdots>\overline{\alpha\left(q_{0}\right)} & \text { if } & c_{n}=1,
\end{array}
$$

because $\alpha\left(q_{0}\right)$ begins with $a_{1} \ldots a_{m}$.

We claim that the word $\omega=c_{1} \cdots c_{N}$ satisfies $\omega\left(\omega_{k}^{-}\right)^{\infty} \in \mathcal{U}_{q_{0}^{*}}^{\prime}$ for all $k \geq 0$. Since $\left(\omega_{k}^{-}\right)^{\infty} \in \mathcal{U}_{q_{0}^{*}}^{\prime}$ by Lemma 2.2, it suffices to prove for each $0<n \leq N$ the relations

$$
\begin{array}{lll}
c_{n+1} \cdots c_{N}\left(\omega_{k}^{-}\right)^{\infty}<\alpha\left(q_{0}^{*}\right) & \text { if } & c_{n}=0, \\
c_{n+1} \cdots c_{N}\left(\omega_{k}^{-}\right)^{\infty}>\overline{\alpha\left(q_{0}^{*}\right)} & \text { if } \quad & c_{n}=1 .
\end{array}
$$

If $n=N$, then $c_{N}=0$ and $\left(\omega_{k}^{-}\right)^{\infty}=\alpha\left(q_{k}\right)<\alpha\left(q_{0}^{*}\right)$. Otherwise the relations follow from (5.7) because each $\left(\omega_{k}^{-}\right)^{\infty}$ begins with $c_{N+1} \cdots c_{N+m-1}$ by (5.6), and $\alpha\left(q_{0}^{*}\right)$ begins with $a_{1} \ldots a_{m-1} a_{m}^{+}$.

If $\left(c_{i}\right)=c_{1} \cdots c_{N}\left(\omega_{0}^{-}\right)^{\infty}$, then it is of the form (5.4). Otherwise there is a largest integer $j_{0} \geq 1$ satisfying $c_{N+1} \cdots c_{N+j_{0} m}=\left(\omega_{0}^{-}\right)^{j_{0}}$. Then by (5.5) and $\left(c_{i}\right) \in \mathcal{U}_{q_{0}^{*}}^{\prime}$ we have

$$
c_{N+j_{0} m+1} \cdots c_{N+\left(j_{0}+1\right) m}=\omega_{0} .
$$

Let $k_{1} \geq 0$ be the largest integer such that $c_{N+j_{0} m+1} \cdots c_{N+j_{0} m+2^{k_{1} m}}=\omega_{k_{1}}$.

Since $c_{N+j_{0} m}=0$ and

$$
c_{N+j_{0} m+1} \cdots c_{N+j_{0} m+2^{k_{1} m}}=\omega_{k_{1}},
$$

by Lemma 5.1 and the maximality of $k_{1}$ the next block of length $2^{k_{1}} \mathrm{~m}$ must be

$$
c_{N+j_{0} m+2^{k_{1}} m+1} \cdots c_{N+j_{0} m+2^{k_{1}+1} m}=\overline{\omega_{k_{1}}} .
$$

Then Lemma 5.1 implies that the next block of length $2^{k_{1}} \mathrm{~m}$ is

$$
c_{N+j_{0} m+2^{k_{1}+1} m+1} \cdots c_{N+j_{0} m+2^{k_{1}+1} m+2^{k_{1}} m}=\omega_{k_{1}} \text { or } \omega_{k_{1}}^{-} .
$$

Hence

$$
c_{N+j_{0} m+1} \cdots c_{N+j_{0} m+2^{k_{1}+1} m+2^{k_{1}} m}=\omega_{k_{1}} \overline{\omega_{k_{1}}} \omega_{k_{1}}
$$

or

$$
c_{N+j_{0} m+1} \cdots c_{N+j_{0} m+2^{k_{1}+1} m+2^{k_{1}} m}=\omega_{k_{1}} \overline{\omega_{k_{1}+1}} .
$$

In the first subcase we either have

$$
c_{N+j_{0} m+1} c_{N+j_{0} m+2} \cdots=\left(\omega_{k_{1}} \overline{\omega_{k_{1}}}\right)^{\infty},
$$

or there exists a largest integer $j_{1} \geq 1$ and a largest integer $k_{2}>k_{1}$ such that

$$
c_{N+j_{0} m+1} \cdots c_{N+j_{0} m+j_{1} 2^{k_{1}+1} m+2^{k_{2} m}}=\left(\omega_{k_{1}} \overline{\omega_{k_{1}}}\right)^{j_{1}} \omega_{k_{2}} .
$$

In the second subcase there exists a largest integer $k_{2}>k_{1}$ such that

$$
c_{N+j_{0} m+1} \cdots c_{N+j_{0} m+2^{k_{1}} m+2^{k_{2}} m}=\omega_{k_{1}} \overline{\omega_{k_{2}}} .
$$

Using (5.8)-(5.10) we see that if $\left(c_{u+i}\right)$ starts with $\omega_{k}$ where $k$ is the largest such index, then three possibilities may occur:

(i) $\left(c_{u+i}\right)=\left(\omega_{k} \overline{\omega_{k}}\right)^{\infty}$;

(ii) $\left(c_{u+i}\right)$ starts with $\left(\omega_{k} \overline{\omega_{k}}\right)^{j_{k}} \omega_{\ell}$ for some $j_{k} \geq 1$ and a maximal $\ell>k$; 
(iii) $\left(c_{u+i}\right)$ starts with $\left(\omega_{k} \overline{\omega_{k}}\right)^{j_{k}} \omega_{k} \overline{\omega_{\ell}}$ for some $j_{k} \geq 0$ and a maximal $\ell>k$.

Repeating the above reasoning in Subcase (ii) for sequences starting with $\omega_{\ell}$, we have three possibilities:

(iia) $\left(c_{u+i}\right)=\left(\omega_{k} \overline{\omega_{k}}\right)^{j_{k}}\left(\omega_{\ell} \overline{\omega_{\ell}}\right)^{\infty}$ for some $j_{k} \geq 1$;

(iib) $\left(c_{u+i}\right)$ starts with $\left(\omega_{k} \overline{\omega_{k}}\right)^{j_{k}}\left(\omega_{\ell} \overline{\omega_{\ell}}\right)^{j_{\ell}} \omega_{n}$ for some $j_{k} \geq 1, j_{\ell} \geq 1$ and a maximal $n>\ell$;

(iic) $\left(c_{u+i}\right)$ starts with $\left(\omega_{k} \overline{\omega_{k}}\right)^{j_{k}}\left(\omega_{\ell} \overline{\omega_{\ell}}\right)^{j_{\ell}} \omega_{\ell} \overline{\omega_{n}}$ for some $j_{k} \geq 1, j_{\ell} \geq 0$ and a maximal $n>\ell$.

Repeating the above reasoning in Subcase (iii) for sequences starting with $\bar{\omega}_{\ell}$, we have three possibilities again:

(iiia) $\left(c_{u+i}\right)=\left(\omega_{k} \overline{\omega_{k}}\right)^{j_{k}} \omega_{k}\left(\overline{\omega_{\ell}} \omega_{\ell}\right)^{\infty}=\left(\omega_{k} \overline{\omega_{k}}\right)^{j_{k}}\left(\omega_{k} \overline{\omega_{\ell}}\right)\left(\omega_{\ell} \overline{\omega_{\ell}}\right)^{\infty}$;

(iiib) $\left(c_{u+i}\right)$ starts with

$$
\left(\omega_{k} \overline{\omega_{k}}\right)^{j_{k}} \omega_{k}\left(\overline{\omega_{\ell}} \omega_{\ell}\right)^{j_{\ell}} \overline{\omega_{n}}=\left(\omega_{k} \overline{\omega_{k}}\right)^{j_{k}}\left(\omega_{k} \overline{\omega_{\ell}}\right)\left(\omega_{\ell} \overline{\omega_{\ell}}\right)^{j_{\ell}-1}\left(\omega_{\ell} \overline{\omega_{n}}\right)
$$

for some $j_{k} \geq 0, j_{\ell} \geq 1$ and a maximal $n>\ell$;

(iiic) $\left(c_{u+i}\right)$ starts with

$$
\left(\omega_{k} \overline{\omega_{k}}\right)^{j_{k}} \omega_{k}\left(\overline{\omega_{\ell}} \omega_{\ell}\right)^{j_{\ell}} \overline{\omega_{\ell}} \omega_{n}=\left(\omega_{k} \overline{\omega_{k}}\right)^{j_{k}}\left(\omega_{k} \overline{\omega_{\ell}}\right)\left(\omega_{\ell} \overline{\omega_{\ell}}\right)^{j_{\ell}} \omega_{n}
$$

for some $j_{k} \geq 0, j_{\ell} \geq 0$ and a maximal $n>\ell$.

Iterating this reasoning in subcases (iib), (iic), (iiib) and (iiic) we obtain eventually that

$$
\left(c_{i}\right)=c_{1} \cdots c_{N}\left(\omega_{0}^{-}\right)^{j_{0}}\left(\omega_{k_{1}} \overline{\omega_{k_{1}}}\right)^{j_{1}}\left(\omega_{k_{1}} \overline{\omega_{k_{2}}}\right)^{s_{2}}\left(\omega_{k_{2}} \overline{\omega_{k_{2}}}\right)^{j_{2}}\left(\omega_{k_{2}} \overline{\omega_{k_{3}}}\right)^{s_{3}} \cdots
$$

with $k_{r}, j_{r}, s_{r}$ as specified in the statement of the theorem.

Now we prove that, conversely, each sequence of the form (5.4) belongs to $\mathcal{U}_{q_{0}^{*}}^{\prime} \backslash \mathcal{U}_{q_{0}}^{\prime}$. Take a sequence $\left(c_{i}\right)$ of form (5.4). By Lemma 2.2 it suffices to prove that

$$
\begin{array}{lll}
c_{n+1} c_{n+2} \ldots<\alpha\left(q_{0}^{*}\right) & \text { whenever } & c_{n}=0, \\
c_{n+1} c_{n+2} \ldots>\overline{\alpha\left(q_{0}^{*}\right)} & \text { whenever } \quad c_{n}=1 .
\end{array}
$$

Write $c_{1} \ldots c_{N}=\omega$ and $\omega_{0}^{-}=a_{1} \ldots a_{m}$. We distinguish three cases.

First case: $0<n<N$. Since $\left(\omega_{k}^{-}\right)^{\infty}$ is strictly increasing as $k \rightarrow \infty$, there exists a large integer $k$ such that

$$
\begin{array}{lll}
c_{n+1} c_{n+2} \ldots \leq c_{n+1} \ldots c_{N}\left(\omega_{k}^{-}\right)^{\infty}<\alpha\left(q_{0}^{*}\right) & \text { if } & c_{n}=0, \\
c_{n+1} c_{n+2} \ldots \geq c_{n+1} \ldots c_{N}\left(\omega_{0}^{-}\right)^{\infty}>\overline{\alpha\left(q_{0}^{*}\right)} & \text { if } \quad c_{n}=1 ;
\end{array}
$$

the second inequality of each line follows from the relations $\omega\left(\omega_{k}^{-}\right)^{\infty} \in \mathcal{U}_{q_{0}^{*}}^{\prime}$ for all $k \geq 0$.

Second case: $N \leq n<N+j_{0} m$. Writing $\omega_{0}^{-}=: a_{1} \ldots a_{m}$ again, we observe that

$$
c_{N+j_{0} m+1} \cdots c_{N+j_{0} m+m}=\omega_{0}=a_{1} \ldots a_{m}^{+},
$$

and therefore

$$
c_{n+1} \cdots c_{n+m}=a_{i+1} \ldots a_{m} a_{1} \ldots a_{i} \text { for some } \quad 0 \leq i<m .
$$

We infer from Lemma 5.2 that

$$
\overline{a_{1} \ldots a_{m-i}} \leq a_{i+1} \ldots a_{m}<a_{1} \ldots a_{m-i} \text { for all } 0<i<m .
$$


Hence

$$
a_{i+1} \ldots a_{m} a_{1} \ldots a_{i}<a_{1} \ldots a_{m}^{+}=\omega_{0} \quad \text { for all } \quad 0 \leq i<m
$$

and

$$
a_{i+1} \ldots a_{m} a_{1} \ldots a_{i} \geq \overline{a_{1} \ldots a_{m}}>\overline{a_{1} \ldots a_{m}^{+}}=\overline{\omega_{0}} \quad \text { for all } \quad 0<i<m .
$$

Since $\alpha\left(q_{0}^{*}\right)$ begins with $\omega_{0}$, in view of (5.12) they imply (5.11).

Third case: $n \geq N+j_{0} m$. Write $\omega_{k}:=\theta_{1} \cdots \theta_{2^{k} m}$ for $k=0,1, \ldots$ By definition $\omega_{k}$ is a prefix of $\alpha\left(q_{0}^{*}\right)$ for all $k \geq 0$. By Lemma 5.2 the following strict inequalities hold for all $k \geq 0$ and $0<i \leq 2^{k} m$ :

$$
\theta_{i+1} \cdots \theta_{2^{k} m} \overline{\theta_{1} \cdots \theta_{i}}<\theta_{1} \cdots \theta_{2^{k} m} \quad \text { and } \quad \theta_{i+1} \cdots \theta_{2^{k} m}>\overline{\theta_{1} \cdots \theta_{2^{k} m-i}} .
$$

Since $\omega_{k}$ is a prefix of $\omega_{\ell}$ whenever $k<\ell$, it follows that $c_{n+1} c_{n+2} \ldots$ satisfies (5.11).

Furthermore, we note that $\left(\omega_{0}^{-}\right)^{\infty} \notin \mathcal{U}_{q_{0}}^{\prime}$ and that the words $\omega_{k}$ are forbidden in $\mathcal{U}_{q_{0}}^{\prime}$ for all $k \geq 0$. We conclude that all sequences of the form (5.4) belong to $\mathcal{U}_{q_{0}^{*}}^{\prime} \backslash \mathcal{U}_{q_{0}}^{\prime}$. By symmetry this completes the proof.

Now we recall that $A_{q}^{\prime}$ is the set of sequences $\left(c_{i}\right) \in \mathcal{U}_{q}^{\prime}$ with $c_{1}=0$. Let $\left(q_{0}, q_{0}^{*}\right)$ be a connected component of $(1,2] \backslash \overline{\mathcal{U}}$ generated by $\omega_{0}^{-}$and write $\left(q_{0}, q_{0}^{*}\right) \cap \mathcal{V}=\left\{q_{n}: n \geq 1\right\}$ as above. Then for each $n \geq 0$ we have $\alpha\left(q_{n}\right)=\left(\omega_{n}^{-}\right)^{\infty}=\left(\omega_{n-1} \overline{\omega_{n-1}}\right)^{\infty}$. We are going to describe $A_{q}^{\prime} \backslash A_{q_{0}}^{\prime}$ for any $q \in\left(q_{n}, q_{n+1}\right]$. This is based on the following lemma:

Lemma 5.5. Let $\left(q_{0}, q_{0}^{*}\right)$ be a connected component of $(1,2] \backslash \overline{\mathcal{U}}$ generated by $\omega_{0}^{-}=a_{1} \ldots a_{m}$. Suppose $\left(q_{0}, q_{0}^{*}\right) \cap \mathcal{V}=\left\{q_{n}: n \geq 1\right\}$. Then for any $q \in\left(q_{n}, q_{n+1}\right]$ the words $\omega_{n}$ and its reflection $\overline{\omega_{n}}$ are forbidden in the language of $\mathcal{U}_{q}^{\prime}$.

Proof. Since $\mathcal{U}_{q}^{\prime}$ is reflection invariant, it suffices to prove that $\omega_{n}$ is forbidden.

Assume on the contrary that there exists a sequence $\left(c_{i}\right) \in \mathcal{U}_{q}^{\prime}$ with $c_{N+1} \cdots c_{N+2^{n} m}=\omega_{n}$. Since $\alpha(q) \leq \alpha\left(q_{n+1}\right)=\left(\omega_{n} \overline{\omega_{n}}\right)^{\infty}$, it follows that $c_{N}=0$, and then $c_{N+1} c_{N+2} \cdots<\left(\omega_{n} \overline{\omega_{n}}\right)^{\infty}$ by Lemma 2.2. This implies that

$$
c_{N+2^{n} m+1} \cdots c_{N+2^{n+1} m} \leq \overline{\omega_{n}} .
$$

On the other hand, since $c_{N+2^{n} m}=1$, we have $c_{N+2^{n} m+1} \cdots c_{N+2^{n+1} m}>\left(\overline{\omega_{n}} \omega_{n}\right)^{\infty}$ by Lemma 2.2. Combining with (5.13) this yields that

$$
c_{N+2^{n} m+1} \cdots c_{N+2^{n+1} m}=\overline{\omega_{n}} .
$$

A similar argument shows that if $c_{u+1} \cdots c_{u+2^{n} m}=\overline{\omega_{n}}$, then the next block of length $2^{n} m$ is $\omega_{n}$.

Iterating the above reasoning we conclude that if $\left(c_{i}\right)$ has a word $\omega_{n}$ then it will eventually end with $\left(\omega_{n} \overline{\omega_{n}}\right)^{\infty}=\alpha\left(q_{n+1}\right)$. This contradicts our initial assumption $\left(c_{i}\right) \in \mathcal{U}_{q}^{\prime}$.

Theorem 5.3 and Lemma 5.5 imply the following

Corollary 5.6. Let $\left(q_{0}, q_{0}^{*}\right)$ be a connected component of $(1,2] \backslash \overline{\mathcal{U}}$ generated by $\omega_{0}^{-}$, and let $\left(q_{0}, q_{0}^{*}\right) \cap \mathcal{V}=\left\{q_{n}: n \geq 1\right\}$ with $q_{1}<q_{2}<\cdots$. If $q \in\left(q_{n}, q_{n+1}\right]$ for some $n \geq 1$, then all elements of $A_{q}^{\prime} \backslash A_{q_{0}}^{\prime}$ are given by

$$
\omega\left(\omega_{0}^{-}\right)^{j_{0}}\left(\omega_{k_{1}} \overline{\omega_{k_{1}}}\right)^{j_{1}}\left(\omega_{k_{1}} \overline{\omega_{k_{2}}}\right)^{s_{2}}\left(\omega_{k_{2}} \overline{\omega_{k_{2}}}\right)^{j_{2}} \cdots\left(\omega_{k_{m-1}} \overline{\omega_{k_{m}}}\right)^{s_{m}}\left(\omega_{k_{m}} \overline{\omega_{k_{m}}}\right)^{j_{m}},
$$

where the nonempty word $\omega$ satisfies $\omega\left(\omega_{k}^{-}\right)^{\infty} \in A_{q}^{\prime}$ for all $0 \leq k<n$, and

$$
\begin{aligned}
& 0 \leq k_{1}<k_{2}<k_{3}<\cdots<k_{m}<n \text { are integers; } \\
& j_{r} \in\{0,1, \cdots\} \text { for } r=0, \ldots, m-1, \text { and } j_{m}=\infty ; \\
& s_{r} \in\{0,1\} \text { for } r=2, \ldots, m .
\end{aligned}
$$




\section{Remark 5.7.}

- We emphasize that the initial word $\omega$ cannot be empty. For otherwise the corresponding sequence might begin with the digit 1 for $j_{0}=0$, while the sequences in $A_{q}^{\prime}$ start with 0 .

- If $\left(q_{0}, q_{0}^{*}\right)=\left(1, q_{K L}\right)$, then the initial word $\omega$ is simply of the form $0^{j}$ because $\left(\omega_{0}^{-}\right)^{\infty}=0^{\infty}$.

\section{Proof of Theorem 1.7 (iv)}

Based on the results of the preceding section, in this section we investigate the derived sets $\mathcal{B}_{2}^{(1)}, \mathcal{B}_{2}^{(2)}, \ldots$

Since $\overline{\mathcal{U}} \subset \mathcal{B}_{2}^{(\infty)}$ by Lemma 4.1 it suffices to investigate the derived sets $\mathcal{B}_{2}^{(j)}$ in each connected component $\left(q_{0}, q_{0}^{*}\right)$ of $(1,2] \backslash \overline{\mathcal{U}}$.

In the following we consider an arbitrary connected component $\left(q_{0}, q_{0}^{*}\right)$ of $(1,2] \backslash \overline{\mathcal{U}}$ generated by $\omega_{0}^{-}=a_{1} \cdots a_{m}$ satisfying (5.2), and we write $\left(q_{0}, q_{0}^{*}\right) \cap \mathcal{V}=\left\{q_{n}: n \geq 1\right\}$ with $q_{1}<q_{2}<\cdots$ as usual, so that

$$
\left(q_{0}, q_{0}^{*}\right)=\bigcup_{n=0}^{\infty}\left(q_{n}, q_{n+1}\right]
$$

By Corollary 5.6 and Lemma 3.2 for each $q \in\left(q_{n}, q_{n+1}\right] \cap \mathcal{B}_{2}$ there exist two words $\omega, \tilde{\omega}$ satisfying $\omega\left(\omega_{k}^{-}\right)^{\infty}, \tilde{\omega}\left(\omega_{k}^{-}\right)^{\infty} \in A_{q}^{\prime}$ for all $0 \leq k<n$, and a pair of vectors

$$
(\mathbf{k}, \mathbf{s}, \mathbf{j}) \text { and }(\tilde{\mathbf{k}}, \tilde{\mathbf{s}}, \tilde{\mathbf{j}})
$$

satisfying the statements in Corollary [5.6] such that $q=q_{\omega, \mathbf{k}, \mathbf{s}, \mathbf{j} ; \tilde{\omega}, \tilde{\mathbf{k}}, \tilde{\mathbf{s}}, \tilde{\mathbf{j}}}$ with an obvious notation. We mention that a $q \in \mathcal{B}_{2}$ may have multiple representations (see Remark 4.2).

We have the following result:

Lemma 6.1. Let $\left(q_{0}, q_{0}^{*}\right)$ be a connected component of $(1,2] \backslash \overline{\mathcal{U}}$ generated by $\omega_{0}^{-}$and write $\left(q_{0}, q_{0}^{*}\right) \cap$ $\mathcal{V}=\left\{q_{n}: n \geq 1\right\}$ with $q_{1}<q_{2}<\cdots$. Suppose

$$
q=q_{\omega, \mathbf{k}, \mathbf{s}, \mathbf{j} ; \tilde{\omega}, \tilde{\mathbf{k}}, \tilde{\mathbf{s}} \tilde{\mathbf{j}}} \in \mathcal{B}_{2} \cap\left(q_{n}, q_{n+1}\right]
$$

for some $n \geq 0$, where the words $\omega, \tilde{\omega}$ satisfy $\omega\left(\omega_{k}^{-}\right)^{\infty} \in A_{q}^{\prime}, \tilde{\omega}\left(\omega_{k}^{-}\right)^{\infty} \in A_{q}^{\prime}$ for all $0 \leq k<n$, and the vectors

$$
\begin{aligned}
(\mathbf{k}, \mathbf{s}, \mathbf{j}) & =\left(k_{1}, \ldots, k_{m_{1}} ; s_{2}, \ldots, s_{m_{1}} ; j_{0}, \cdots, j_{m_{1}-1}, \infty\right) \\
(\tilde{\mathbf{k}}, \tilde{\mathbf{s}}, \tilde{\mathbf{j}}) & =\left(\tilde{k}_{1}, \ldots, \tilde{k}_{m_{2}} ; \tilde{s}_{2}, \ldots, \tilde{s}_{m_{2}} ; \tilde{j}_{0}, \cdots, \tilde{j}_{m_{2}-1}, \infty\right)
\end{aligned}
$$

satisfy for some $j \geq 0$ the conditions

$$
m_{1} \geq 0, \quad m_{2} \geq 0 \quad \text { and } \quad 0 \leq\left(k_{m_{1}}+1\right)+\left(\tilde{k}_{m_{2}}+1\right) \leq 2 n-j .
$$

(Here we use the convention that $k_{m_{1}}:=-1$ if $m_{1}=0$ and $\tilde{k}_{m_{2}}=-1$ if $m_{2}=0$.) Then $q \in\left(\mathcal{B}_{2} \cap\left(q_{n}, q_{n+1}\right]\right)^{(j)}$.

Moreover, in case $j \geq 1$ and $q \in \mathcal{B}_{2}^{(j)} \cap\left(q_{n}, q_{n+1}\right)$ then there exist two sequences $\left(p_{u}\right),\left(r_{u}\right)$ in $\mathcal{B}_{2}^{(j-1)} \cap\left(q_{n}, q_{n+1}\right)$ such that $p_{u} \nearrow q$ and $r_{u} \searrow q$ as $u \rightarrow \infty$.

Proof. For $j=0$ the lemma follows from Corollary 5.6 and Lemma 3.2. Proceeding by induction on $j$, assume that the assertion is true for some $0 \leq j<2 n$, and consider $j+1$. 
Take a point $q_{\omega, \mathbf{k}, \mathbf{s}, \mathbf{j} ; \tilde{\omega}, \tilde{\mathbf{k}}, \tilde{\mathbf{s}}, \tilde{\mathbf{j}}} \in \mathcal{B}_{2} \cap\left(q_{n}, q_{n+1}\right]$ such that $\omega\left(\omega_{k}^{-}\right)^{\infty}, \tilde{\omega}\left(\omega_{k}^{-}\right)^{\infty} \in A_{q}^{\prime}$ for all $0 \leq k<n$, and

$$
\begin{aligned}
(\mathbf{k}, \mathbf{s}, \mathbf{j}) & =\left(k_{1}, \ldots, k_{m_{1}} ; s_{2}, \ldots, s_{m_{1}} ; j_{0}, \cdots, j_{m_{1}-1}, \infty\right) \\
(\tilde{\mathbf{k}}, \tilde{\mathbf{s}}, \tilde{\mathbf{j}}) & =\left(\tilde{k}_{1}, \ldots, \tilde{k}_{m_{2}} ; \tilde{s}_{2}, \ldots, \tilde{s}_{m_{2}} ; \tilde{j}_{0}, \cdots, \tilde{j}_{m_{2}-1}, \infty\right)
\end{aligned}
$$

satisfy

$$
0 \leq\left(k_{m_{1}}+1\right)+\left(\tilde{k}_{m_{2}}+1\right) \leq 2 n-(j+1) .
$$

We will show that $q_{\omega, \mathbf{k}, \mathbf{s}, \mathbf{j} ; \tilde{\omega}, \tilde{\mathbf{k}}, \tilde{\mathbf{s}}, \tilde{\mathbf{j}}} \in\left(\mathcal{B}_{2} \cap\left(q_{n}, q_{n+1}\right]\right)^{(j+1)}$ by distinguishing the cases

$$
q_{\omega, \mathbf{k}, \mathbf{s}, \mathbf{j} ; \tilde{\omega}, \tilde{\mathbf{k}}, \tilde{\mathbf{s}}, \tilde{\mathbf{j}}} \in\left(q_{n}, q_{n+1}\right) \quad \text { and } \quad q_{\omega, \mathbf{k}, \mathbf{s}, \mathbf{j} ; \tilde{\omega}, \tilde{\mathbf{k}}, \tilde{\mathbf{s}}, \tilde{\mathbf{j}}}=q_{n+1} .
$$

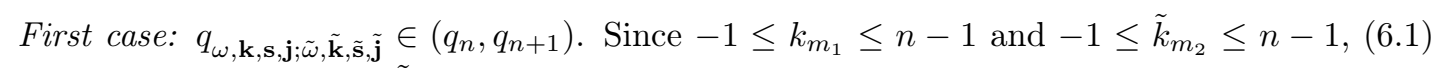
implies that $k_{m_{1}}<n-1$ or $\tilde{k}_{m_{2}}<n-1$. Without loss of generality we assume $k_{m_{1}}<n-1$, and we consider for each $u \geq 1$ the vectors

$$
\left(\mathbf{k}^{+}, \mathbf{s}^{-}, \mathbf{j}_{u}\right):=\left(k_{1}, \ldots, k_{m_{1}}, k_{m_{1}}+1 ; s_{2}, \ldots, s_{m_{1}}, 0 ; j_{0}, \cdots, j_{m_{1}-1}, u, \infty\right)
$$

and

$$
\left(\mathbf{k}^{+}, \mathbf{s}^{+}, \mathbf{j}_{u}\right):=\left(k_{1}, \ldots, k_{m_{1}}, k_{m_{1}}+1 ; s_{2}, \ldots, s_{m_{1}}, 1 ; j_{0}, \cdots, j_{m_{1}-1}, u, \infty\right) .
$$

Applying the induction hypothesis, it follows by continuity that

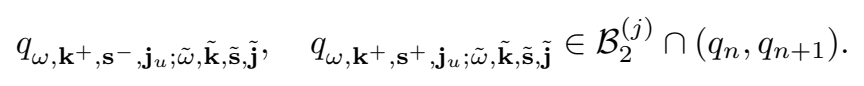

for all sufficiently large $u$. Writing

$$
\begin{aligned}
& \omega^{\mathbf{k}, \mathbf{s}, \mathbf{j}}:=\left(\omega_{0}^{-}\right)^{j_{0}}\left(\omega_{k_{1}} \overline{\omega_{k_{1}}}\right)^{j_{1}}\left(\omega_{k_{1}} \overline{\omega_{k_{2}}}\right)^{s_{2}}\left(\omega_{k_{2}} \overline{\omega_{k_{2}}}\right)^{j_{2}} \cdots\left(\omega_{k_{m_{1}-1}} \overline{\omega_{k_{m_{1}}}}\right)^{s_{m_{1}}}\left(\omega_{k_{m_{1}}} \overline{\omega_{k_{m_{1}}}}\right)^{\infty}, \\
& \omega^{\mathbf{k}^{+}, \mathbf{s}^{-}, \mathbf{j}_{u}}:=\left(\omega_{0}^{-}\right)^{j_{0}}\left(\omega_{k_{1}} \overline{\omega_{k_{1}}}\right)^{j_{1}}\left(\omega_{k_{1}} \overline{\omega_{k_{2}}}\right)^{s_{2}}\left(\omega_{k_{2}} \overline{\omega_{k_{2}}}\right)^{j_{2}} \ldots \\
& \left(\omega_{k_{m_{1}-1}} \overline{\omega_{k_{m_{1}}}}\right)^{s_{m_{1}}}\left(\omega_{k_{m_{1}}} \overline{\omega_{k_{m_{1}}}}\right)^{u}\left(\omega_{k_{m_{1}}+1} \overline{\omega_{k_{m_{1}}+1}}\right)^{\infty} \\
& \omega^{\mathbf{k}^{+}, \mathbf{s}^{+}, \mathbf{j}_{u}}:=\left(\omega_{0}^{-}\right)^{j_{0}}\left(\omega_{k_{1}} \overline{\omega_{k_{1}}}\right)^{j_{1}}\left(\omega_{k_{1}} \overline{\omega_{k_{2}}}\right)^{s_{2}}\left(\omega_{k_{2}} \overline{\omega_{k_{2}}}\right)^{j_{2}} \ldots \\
& \left(\omega_{k_{m_{1}-1}} \overline{\omega_{k_{m_{1}}}}\right)^{s_{m_{1}}}\left(\omega_{k_{m_{1}}} \overline{\omega_{k_{m_{1}}}}\right)^{u}\left(\omega_{k_{m_{1}}} \overline{\omega_{k_{m_{1}}+1}}\right)\left(\omega_{k_{m_{1}+1}} \overline{\omega_{k_{m_{1}}+1}}\right)^{\infty}
\end{aligned}
$$

for convenience, we have

$$
\omega^{\mathbf{k}^{+}, \mathbf{s}^{-}, \mathbf{j}_{1}}>\omega^{\mathbf{k}^{+}, \mathbf{s}^{-}, \mathbf{j}_{2}}>\omega^{\mathbf{k}^{+}, \mathbf{s}^{-}, \mathbf{j}_{3}}>\cdots>\omega^{\mathbf{k}, \mathbf{s}, \mathbf{j}}
$$

and

$$
\omega^{\mathbf{k}^{+}, \mathbf{s}^{+}, \mathbf{j}_{1}}<\omega^{\mathbf{k}^{+}, \mathbf{s}^{+}, \mathbf{j}_{2}}<\omega^{\mathbf{k}^{+}, \mathbf{s}^{+}, \mathbf{j}_{3}}<\cdots<\omega^{\mathbf{k}, \mathbf{s}, \mathbf{j}} .
$$

Therefore, using Lemma 3.5 we obtain that for sufficiently large $u$ the sequences

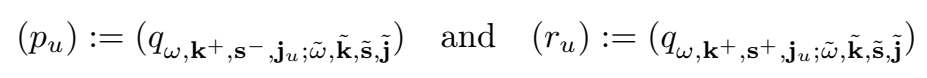

belong to $\mathcal{B}_{2}^{(j)} \cap\left(q_{n}, q_{n+1}\right)$ and

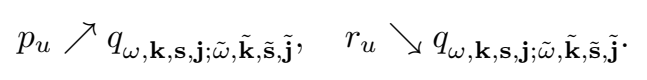

In particular, $q_{\omega, \mathbf{k}, \mathbf{s}, \mathbf{j} ; \tilde{\omega}, \tilde{\mathbf{k}}, \tilde{\mathbf{s}}, \tilde{\mathbf{j}}} \in\left(\mathcal{B}_{2} \cap\left(q_{n}, q_{n+1}\right]\right)^{(j+1)}$.

Second case: $q_{\omega, \mathbf{k}, \mathbf{s}, \mathbf{j} ; \tilde{\omega}, \tilde{\mathbf{k}}, \tilde{\mathbf{s}}, \tilde{\mathbf{j}}}=q_{n+1}$. We obtain similarly to the preceding case that there exists a sequence $\left(p_{u}\right) \in\left(\mathcal{B}_{2} \cap\left(q_{n}, q_{n+1}\right]\right)^{(j)}$ satisfying $p_{u} \nearrow q_{\omega, \mathbf{k}, \mathbf{s}, \mathbf{j} ; \tilde{\omega}, \tilde{\mathbf{k}}, \tilde{\mathbf{s}}, \tilde{\mathbf{j}}}$ as $u \rightarrow \infty$. Therefore, $q_{\omega, \mathbf{k}, \mathbf{s}, \mathbf{j} ; \tilde{\omega}, \tilde{\mathbf{k}}, \tilde{\mathbf{s}}, \tilde{\mathbf{j}}} \in\left(\mathcal{B}_{2} \cap\left(q_{n}, q_{n+1}\right]\right)^{(j+1)}$ as required. 
Proposition 6.2. Let $\left(q_{0}, q_{0}^{*}\right)$ be a connected component of $(1,2] \backslash \overline{\mathcal{U}}$ generated by $\omega_{0}^{-}=a_{1} \ldots a_{m}$ and write $\left(q_{0}, q_{0}^{*}\right) \cap \mathcal{V}=\left\{q_{n}: n \geq 1\right\}$ with $q_{1}<q_{2}<\cdots$. Then

$$
\mathcal{B}_{2}^{(n)} \cap\left(q_{n}, q_{n+1}\right) \neq \emptyset \quad \text { for all } \quad n \geq 2 .
$$

Remark 6.3. The assumption $n \geq 2$ cannot be omitted. Indeed, for $\left(q_{0}, q_{0}^{*}\right)=\left(1, q_{K L}\right)$ we know that $\mathcal{B}_{2} \cap\left(q_{0}, q_{2}\right)=\left\{q_{s}\right\}$. Since $q_{s}>q_{1}$, this implies that

$$
\mathcal{B}_{2} \cap\left(q_{0}, q_{1}\right)=\mathcal{B}_{2}^{(1)} \cap\left(q_{1}, q_{2}\right)=\emptyset .
$$

ProOF. Let $n \geq 2$, and set

$$
\omega=0^{2^{n} m} \overline{\omega_{0}}, \quad \tilde{\omega}=\overline{\omega_{0}},
$$

and

$$
(\mathbf{k}, \mathbf{s}, \mathbf{j})=(0 ; \emptyset ; 1, \infty), \quad(\tilde{\mathbf{k}}, \tilde{\mathbf{s}}, \tilde{\mathbf{j}})=(0,1, \ldots, n-2 ; 0, \ldots, 0 ; 1, \ldots, 1, \infty) .
$$

Note that $\omega_{0}=a_{1} \cdots a_{m}^{+}$. Take $q \in\left(q_{n}, q_{n+1}\right)$ with $n \geq 2$. Then $\alpha(q)$ begins with $a_{1} \ldots a_{m}^{+} \overline{a_{1} \ldots a_{m}}$. Pick $0 \leq k<n$. Using Lemma 5.2 we see that

$$
\left.\sigma^{i} \overline{\left(a_{1} \cdots a_{m}^{+}\right.}\left(\omega_{k}^{-}\right)^{\infty}\right)<a_{1} \cdots a_{m}^{+} \overline{a_{1} \cdots a_{m}} \ldots=\alpha(q)
$$

and

$$
\left.\sigma^{i} \overline{\left(a_{1} \cdots a_{m}^{+}\right.}\left(\omega_{k}^{-}\right)^{\infty}\right)>\overline{a_{1} \cdots a_{m}^{+}} a_{1} \cdots a_{m} \ldots=\overline{\alpha(q)}
$$

for all $i \geq 0$. Hence $\overline{\omega_{0}}\left(\omega_{k}^{-}\right)^{\infty} \in A_{q}^{\prime}$, and therefore $\omega\left(\omega_{k}^{-}\right)^{\infty}, \tilde{\omega}\left(\omega_{k}^{-}\right)^{\infty} \in A_{q}^{\prime}$ for all $0 \leq k<n$. It follows from Corollary 5.6 that the sequences

$$
\mathbf{c}:=\omega \omega^{\mathbf{k}, \mathbf{s}, \mathbf{j}}=0^{2^{n} m} \overline{\omega_{0}} \omega_{0}^{-}\left(\omega_{0} \overline{\omega_{0}}\right)^{\infty}
$$

and

$$
\mathbf{d}:=\tilde{\omega} \omega^{\tilde{\mathbf{k}}, \tilde{\mathbf{s}}, \tilde{\mathbf{j}}}=\overline{\omega_{0}} \omega_{0}^{-}\left(\omega_{0} \overline{\omega_{0}}\right) \cdots\left(\omega_{n-3} \overline{\omega_{n-3}}\right)\left(\omega_{n-2} \overline{\omega_{n-2}}\right)^{\infty}
$$

also belong to $A_{q}^{\prime}$. We are going to prove that $f_{\mathbf{c}, \mathbf{d}}\left(q_{n}\right)<0$ and $f_{\mathbf{c}, \mathbf{d}}\left(q_{n+1}\right)>0$. It will then follow by Lemmas 3.2 and 3.4 that $f_{\mathbf{c}, \mathbf{d}}$ has a (unique) zero $q_{\omega, \mathbf{k}, \mathbf{s}, \mathbf{j} ; \tilde{\omega}, \tilde{\mathbf{k}}, \tilde{\mathbf{s}}, \tilde{\mathbf{j}}}$ in $\left(q_{n}, q_{n+1}\right)$, and then Lemma 6.1 will imply that $q_{\omega, \mathbf{k}, \mathbf{s}, \mathbf{j} ; \tilde{\omega}, \tilde{\mathbf{k}}, \tilde{\mathbf{s}}, \tilde{\mathbf{j}}} \in \mathcal{B}_{2}^{(n)} \cap\left(q_{n}, q_{n+1}\right)$.

It remains to prove the two inequalities. Since $\overline{\omega_{0}} \omega_{0}^{-}=\overline{\omega_{1}}$ and $\omega_{k} \overline{\omega_{k}}=\omega_{k+1}^{-}$for all $k \geq 0$, we have

$$
\begin{aligned}
f_{\mathbf{c}, \mathbf{d}}(q)= & (1 \mathbf{c})_{q}-(0 \overline{\mathbf{d}})_{q} \\
= & \left(10^{2^{n} m} \overline{\omega_{0}} \omega_{0}^{-}\left(\omega_{0} \overline{\omega_{0}}\right)^{\infty}\right)_{q}-\left(0 \omega_{0} \bar{\omega}_{0}+\left(\overline{\omega_{0}} \omega_{0}\right) \cdots\left(\overline{\omega_{n-3}} \omega_{n-3}\right)\left(\overline{\omega_{n-2}} \omega_{n-2}\right)^{\infty}\right)_{q} \\
= & \left(10^{2^{n} m} \overline{\omega_{1}}\left(\omega_{1}^{-}\right)^{\infty}\right)_{q}-\left(0 \omega_{1} \overline{\omega_{1}}+\overline{\omega_{2}}+\ldots \overline{\omega_{n-2}}+\left(\overline{\omega_{n-1}}\right)^{\infty}\right)_{q} \\
& \vdots \\
= & \left(10^{2^{n} m} \overline{\omega_{1}}\left(\omega_{1}^{-}\right)^{\infty}\right)_{q}-\left(0 \omega_{n}\left(\overline{\omega_{n-1}}\right)^{\infty}\right)_{q}
\end{aligned}
$$

for all $q$. Since $\left(\omega_{n} 0^{\infty}\right)_{q_{n}}=1$ and the word $\omega_{n}$ is of length $2^{n} m$, we infer from (6.2) that

$$
f_{\mathbf{c}, \mathbf{d}}\left(q_{n}\right)=\left(0^{2^{n} m+1} \overline{\omega_{1}}\left(\omega_{1}^{-}\right)^{\infty}\right)_{q_{n}}-\left(0^{2^{n} m+1}\left({\overline{\omega_{n-1}}}^{+}\right)^{\infty}\right)_{q_{n}}<0,
$$

because

$$
\overline{\omega_{1}}\left(\omega_{1}^{-}\right)^{\infty}<\left({\overline{\omega_{n-1}}}^{+}\right)^{\infty}
$$


for all $n \geq 2$. Indeed, $\left({\overline{\omega_{n-1}}}^{+}\right)^{\infty}$ starts with

$$
\begin{aligned}
& \overline{\omega_{1}}>\overline{\omega_{1}} \quad \text { if } \quad n=2, \\
& \overline{\omega_{1}} \omega_{1}>\overline{\omega_{1}} \omega_{1}^{-} \quad \text { if } \quad n=3, \\
& \overline{\omega_{1}} \omega_{1}^{-} \omega_{1}>\overline{\omega_{1}}\left(\omega_{1}^{-}\right)^{2} \quad \text { if } \quad n \geq 4 .
\end{aligned}
$$

Next we observe that $\left(\omega_{n}{\overline{\omega_{n}}}^{+} 0^{\infty}\right)_{q_{n+1}}=1$. Therefore, using (6.2) we have

$$
\begin{aligned}
f_{\mathbf{c}, \mathbf{d}}\left(q_{n+1}\right) & =\left(10^{2^{n} m} \overline{\omega_{1}}\left(\omega_{1}^{-}\right)^{\infty}\right)_{q_{n+1}}-\left(0 \omega_{n}\left({\overline{\omega_{n-1}}}^{+}\right)^{\infty}\right)_{q_{n+1}} \\
& =\left(0^{2^{n} m+1} \overline{\omega_{1}}\left(\omega_{1}^{-}\right)^{\infty}\right)_{q_{n+1}}+\left(0^{2^{n} m+1} \bar{\omega}_{n}^{+} 0^{\infty}\right)_{q_{n+1}}-\left(0^{2^{n} m+1}\left(\overline{\omega_{n-1}}+\right)^{\infty}\right)_{q_{n+1}} \\
& =q^{-2^{n} m-1}\left[\left(\bar{\omega}_{1}\left(\omega_{1}^{-}\right)^{\infty}\right)_{q_{n+1}}+\left(\bar{\omega}_{n}+0^{\infty}\right)_{q_{n+1}}-\left(\left(\bar{\omega}_{n-1}\right)^{\infty}\right)_{q_{n+1}}\right] .
\end{aligned}
$$

We observe that

$$
\left({\overline{\omega_{n-1}}}^{+}\right)^{\infty}=\left(\overline{\omega_{n-2}} \omega_{n-2}\right)^{\infty}=\overline{\omega_{n-2}}\left(\omega_{n-1}^{-}\right)^{\infty}<{\overline{\omega_{n-2}}}^{+} 0^{\infty}
$$

and

$$
{\overline{\omega_{n}}}^{+} 0^{\infty}=\overline{\omega_{n-2}} \omega_{n-2}^{-} \omega_{n-2}{\overline{\omega_{n-2}}}^{+} 0^{\infty}
$$

hence

$$
\left({\overline{\omega_{n-1}}}^{+}\right)^{\infty}<{\overline{\omega_{n}}}^{+} 0^{\infty}+0^{2^{n-2} m-1} 10^{\infty} .
$$

If $n \geq 3$, then

$$
0^{2^{n-2} m-1} 10^{\infty} \leq \overline{\omega_{1}}\left(\omega_{1}^{-}\right)^{\infty}
$$

so that

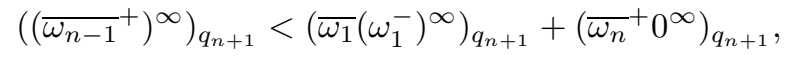

and thus $f_{\mathbf{c}, \mathbf{d}}\left(q_{n+1}\right)>0$ as required.

The relation (6.3) and hence the proof of $f_{\mathbf{c}, \mathbf{d}}\left(q_{n+1}\right)>0$ remains valid if $n=2$ and $\omega_{0}$ contains at least one zero digit.

In the remaining case we have $n=2$ and

$$
\omega_{0}=1^{m}, \quad \omega_{1}=1^{m} 0^{m-1} 1, \quad \omega_{2}=1^{m} 0^{m-1} 10^{m} 1^{m}
$$

for some $m \geq 1$.

If $m \geq 2$, then

$$
\begin{aligned}
\left(\overline{\omega_{1}}\left(\omega_{1}^{-}\right)^{\infty}\right)_{q_{3}}+\left(\overline{\omega_{2}}+0^{\infty}\right)_{q_{3}} & =\left(\left(0^{m} 1^{m-1} 0\left(1^{m} 0^{m}\right)^{\infty}\right)_{q_{3}}+\left(0^{m} 1^{m-1} 01^{m} 0^{m-1} 10^{\infty}\right)_{q_{3}}\right. \\
& \geq\left(0^{m} 10^{\infty}\right)_{q_{3}}+\left(0^{m} 10^{\infty}\right)_{q_{3}} \\
& >\left(0^{m-1} 10^{\infty}\right)_{q_{3}} \\
& >\left(\left(0^{m} 1^{m}\right)^{\infty}\right)_{q_{3}} \\
& =\left(\left(\bar{\omega}_{1}^{+}\right)^{\infty}\right)_{q_{3}},
\end{aligned}
$$

so that (6.4) holds again.

Finally, if $m=1$, then (6.4) takes the form

$$
\left((01)^{\infty}\right)_{q_{3}}<\left(00(10)^{\infty}\right)_{q_{3}}+\left(00110^{\infty}\right)_{q_{3}} .
$$

This is equivalent to

$$
q_{3}^{3}<\left(q_{3}+1\right)^{2},
$$

and this holds because the unique positive root of the polynomial $x^{3}-(x+1)^{2}$ is greater than 2 . 
Proof of Theorem 1.7 (IV). It follows from Proposition 6.2 that

$$
\mathcal{B}_{2}^{(j)} \cap\left(q_{0}, q_{0}^{*}\right) \neq \emptyset \text { for all } j \geq 0
$$

for all connected components $\left(q_{0}, q_{0}^{*}\right)$ of $(1,2] \backslash \overline{\mathcal{U}}$. This implies that all sets $\mathcal{B}_{2}, \mathcal{B}_{2}^{(1)}, \mathcal{B}_{2}^{(2)}, \cdots$ have infinitely many accumulation points in $\left(q_{0}, q_{0}^{*}\right)$.

\section{Proof of Theorem 1.5 (v)-(vi) and Theorem 1.7 (v)-(vi)}

In this section we focus on the derived sets of $\mathcal{B}_{2}$ in the first connected component $\left(1, q_{K L}\right)$ of $(1,2] \backslash \overline{\mathcal{U}}$, generated by $\omega_{0}^{-}=0$.

Note that

$$
\left(1, q_{K L}\right) \backslash \mathcal{V}=\bigcup_{n=0}^{\infty}\left(q_{n}, q_{n+1}\right)
$$

where the numbers $q_{n}$ satisfy

$$
\alpha\left(q_{n}\right)=\left(\omega_{n}^{-}\right)^{\infty}=\left(\tau_{1} \cdots \tau_{2^{n}}^{-}\right)^{\infty}, \quad \beta\left(q_{n}\right)=\omega_{n} 0^{\infty}=\tau_{1} \cdots \tau_{2^{n}} 0^{\infty} \quad \text { and } \quad q_{n} \nearrow q_{K L} \approx 1.78723 .
$$

Here

$$
\alpha\left(q_{K L}\right)=\tau_{1} \tau_{2} \cdots=1101001100101101 \cdots
$$

is the truncated Thue-Morse sequence. We recall that the complete Thue-Morse sequence $\left(\tau_{i}\right)_{i=0}^{\infty}$ is defined by the formulas $\tau_{0}:=0$ and

$$
\tau_{2 i}:=\tau_{i}, \quad \tau_{2 i+1}=1-\tau_{i}, \quad i=0,1, \ldots
$$

The first five elements of the sequence $\left(q_{n}\right)$ are the following:

$$
q_{0}=1, \quad q_{1} \approx 1.61803, \quad q_{2} \approx 1.75488, \quad q_{3} \approx 1.78460, \quad q_{4} \approx 1.78721 .
$$

Note that $q_{1}=\varphi$ is the Golden Ratio and $q_{2}=q_{f}$ is the smallest accumulation point of $\mathcal{B}_{2}$ by Theorem 1.5.

Since $A_{q_{0}}^{\prime}=\emptyset$ for $q_{0}=1$, Corollary 5.6 and Remark 5.8 yields the following description of $A_{q}^{\prime}$ for any $q \in\left(1, q_{K L}\right)$ :

Corollary 7.1. If $q \in\left(q_{n}, q_{n+1}\right] \subset\left(1, q_{K L}\right)$ for some $n \geq 0$, then the elements of $A_{q}^{\prime}$ are given by the formula

$$
0^{j_{0}}\left(\omega_{k_{1}} \overline{\omega_{k_{1}}}\right)^{j_{1}}\left(\omega_{k_{1}} \overline{\omega_{k_{2}}}\right)^{s_{2}}\left(\omega_{k_{2}} \overline{\omega_{k_{2}}}\right)^{j_{2}} \cdots\left(\omega_{k_{m-1}} \overline{\omega_{k_{m}}}\right)^{s_{m}}\left(\omega_{k_{m}} \overline{\omega_{k_{m}}}\right)^{j_{m}},
$$

where

$$
\begin{aligned}
& 0 \leq k_{1}<k_{2}<k_{3}<\cdots<k_{m}<n \text { are integers; } \\
& j_{0} \geq 1, \quad j_{r} \in\{0,1, \cdots\} \text { for } \quad r=0, \ldots, m-1, \text { and } j_{m}=\infty ; \\
& s_{r} \in\{0,1\} \quad \text { for } \quad r=2, \ldots, m .
\end{aligned}
$$

\section{Examples 7.2.}

(i) If $q \in\left(q_{0}, q_{1}\right] \approx(1,1.61803]$, then $n=0$, then

$$
A_{q}^{\prime}=\left\{0^{\infty}\right\} .
$$

(ii) If $q \in\left(q_{1}, q_{2}\right] \approx(1.61803,1.75488]$, then

$$
A_{q}^{\prime}=\left\{0^{\infty}, 0^{j_{0}}(10)^{\infty}: j_{0}=1,2, \ldots\right\} .
$$


(iii) If $q \in\left(q_{2}, q_{3}\right]$, then

$$
A_{q}^{\prime}=\left\{0^{\infty}, 0^{j_{0}}(10)^{\infty}, 0^{j_{0}}(10)^{j_{1}}(100)^{s_{0}}(1100)^{\infty}: j_{0}=1,2, \ldots ; j_{1}=0,1, \ldots ; s_{0} \in\{0,1\}\right\} .
$$

Using Corollary 7.1 we may strengthen a result of Sidorov [27]:

Proposition 7.3. Each $q \in \mathcal{B}_{2} \cap\left(1, q_{K L}\right)$ is an algebraic integer. Hence $\mathcal{B}_{2} \cap\left(1, q_{K L}\right)$ is a countable set.

Proof. If $q \in \mathcal{B}_{2} \cap\left(1, q_{K L}\right)$, then there exist $\left(c_{i}\right),\left(d_{i}\right) \in \mathcal{U}_{q}^{\prime}$ satisfying $\left(1\left(c_{i}\right)\right)_{q}=\left(0\left(d_{i}\right)\right)_{q}$ by Lemma 3.1. Since the sequences $\left(c_{i}\right),\left(d_{i}\right)$ are eventually periodic by Corollary 7.1 , the last equality takes the form

$$
\frac{1}{q}+\frac{f_{1}(q)}{g_{1}(q)}=\frac{f_{2}(q)}{g_{2}(q)}
$$

with suitable polynomials $f_{1}, f_{2}, g_{1}, g_{2}$ of integer coefficients, satisfying the conditions

$$
\operatorname{deg} f_{1} \leq\left(\operatorname{deg} g_{1}\right)-2 \quad \text { and } \quad \operatorname{deg} f_{2} \leq\left(\operatorname{deg} g_{2}\right)-2 .
$$

Furthermore, $g_{1}, g_{2}$ are of the form $q^{m}\left(q^{n}-1\right)$ with suitable positive integers $m, n$. Hence $q$ is a zero of the polynomial

$$
g_{1}(x) g_{2}(x)+x f_{1}(x) g_{2}(x)-x f_{2}(x) g_{1}(x) .
$$

We conclude by observing that this polynomial is monic by the special form of $g_{1}, g_{2}$, mentioned above. vector

If $q \in\left(q_{n}, q_{n+1}\right]$, then by Corollary 7.1 each sequence $\left(c_{i}\right) \in A_{q}^{\prime}$ is uniquely determined by a

$$
(\mathbf{k}, \mathbf{s}, \mathbf{j})=\left(k_{1}, k_{2}, \ldots, k_{m} ; s_{2}, s_{3}, \ldots, s_{m} ; j_{0}, j_{1}, \ldots, j_{m}\right)
$$

with the components as in the statement of Corollary 7.1. Observe that $(\mathbf{k}, \mathbf{s}, \mathbf{j})$ has $3 m$ components if $m \geq 1$, while $(\mathbf{k}, \mathbf{s}, \mathbf{j})=(\infty)$ if $m=0$.

For convenience we set

$$
\omega^{\mathbf{k}, \mathbf{s}, \mathbf{j}}:=0^{j_{0}}\left(\omega_{k_{1}} \overline{\omega_{k_{1}}}\right)^{j_{1}}\left(\omega_{k_{1}} \overline{\omega_{k_{2}}}\right)^{s_{2}}\left(\omega_{k_{2}} \overline{\omega_{k_{2}}}\right)^{j_{2}} \cdots\left(\omega_{k_{m-1}} \overline{\omega_{k_{m}}}\right)^{s_{m}}\left(\omega_{k_{m}} \overline{\omega_{k_{m}}}\right)^{\infty} .
$$

When $(\mathbf{k}, \mathbf{s}, \mathbf{j})=(\infty)$ we set $\omega^{\mathbf{k}, \mathbf{s}, \mathbf{j}}=0^{\infty}$. We infer from Lemma 3.2 that for each $q \in \mathcal{B}_{2} \cap\left(q_{n}, q_{n+1}\right]$ there exists a pair of vectors

$$
(\mathbf{k}, \mathbf{s}, \mathbf{j}) \text { and }(\tilde{\mathbf{k}}, \tilde{\mathbf{s}}, \tilde{\mathbf{j}})
$$

such that setting $\mathbf{c}=\omega^{\mathbf{k}, \mathbf{s}, \mathbf{j}}, \mathbf{d}=\omega^{\tilde{\mathbf{k}}, \tilde{\mathbf{s}}, \tilde{\mathbf{j}}} \in A_{q_{n+1}}^{\prime}$ the equality $f_{\mathbf{c}, \mathbf{d}}(q)=(1 \mathbf{c})_{q}+(1 \mathbf{d})_{q}-\left(1^{\infty}\right)_{q}=0$ holds. In this cases we denote $q$ by $q_{\mathbf{k}, \mathbf{s}, \mathbf{j} ; \tilde{\mathbf{k}}, \tilde{s}, \tilde{\mathbf{j}}}$.

Remark 7.4. We do not rule out the possibility that an element $q \in \mathcal{B}_{2} \cap\left(q_{n}, q_{n+1}\right]$ has multiple representations by different pairs of vectors $(\mathbf{k}, \mathbf{s}, \mathbf{j})$ and $(\tilde{\mathbf{k}}, \tilde{\mathbf{s}}, \tilde{\mathbf{j}})$. See Lemma 7.7 below and Question 6 at the end of the paper.

In order to investigate the topology of $\mathcal{B}_{2} \cap\left(q_{n}, q_{n+1}\right]$ we need the following elementary result, probably first published by Kürschák [24]:

\section{Lemma 7.5.}

(i) Every sequence of real numbers has a monotone subsequence.

(ii) Every sequence $\left(c_{j}\right)$ in $\{0,1, \ldots, \infty\}$ has either a strictly increasing or a constant subsequence. 
Proof. (i) See, e.g., [17, Theorem 1.1 (a), p. 6].

(ii) If $\left(c_{j}\right)$ does not have a constant subsequence, then $\infty$ occurs at most finitely many times, and therefore $\left(c_{j}\right)$ has a monotone subsequence $\left(c_{j_{k}}\right)$. Since $\left(c_{j_{k}}\right)$ has no constant subsequences either, it has a strictly monotone further subsequence. It is necessarily increasing because there is no strictly decreasing sequence of nonnegative integers.

Lemma 7.6. If a sequence

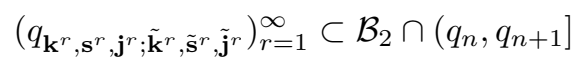

converges to some point $p \in\left[q_{n}, q_{n+1}\right]$ with $n \geq 0$, then there exists a subsequence in which each component sequence is either constant or strictly increasing.

Proof. Observe that there are only finitely many possibilities for the vectors $\mathbf{k}^{r}, \mathbf{s}^{r}, \tilde{\mathbf{k}}^{r}$ and $\tilde{\mathbf{s}}^{r}$. Then there exists a subsequence $\left(r_{i}\right)$ such that

$$
\begin{aligned}
\left(\mathbf{k}^{r_{i}}, \mathbf{s}^{r_{i}}, \mathbf{j}^{r_{i}}\right) & =\left(k_{1}, k_{2}, \ldots, k_{m_{1}} ; s_{2}, s_{3}, \ldots, s_{m_{1}} ; j_{0}^{r_{i}}, j_{1}^{r_{i}}, \ldots, j_{m_{1}-1}^{r_{i}}, \infty\right) ; \\
\left(\tilde{\mathbf{k}}^{r_{i}}, \tilde{\mathbf{s}}^{r_{i}}, \tilde{\mathbf{j}}^{r_{i}}\right) & =\left(\tilde{k}_{1}, \tilde{k}_{2}, \ldots, \tilde{k}_{m_{2}} ; \tilde{s}_{2}, \tilde{s}_{3}, \ldots, \tilde{s}_{m_{2}} ; \tilde{j}_{0}^{r_{i}}, \tilde{j}_{1}^{r_{i}}, \ldots, \tilde{j}_{m_{2}-1}^{r_{i}}, \infty\right)
\end{aligned}
$$

for all $i \geq 1$, where $m_{1}, m_{2} \in\{0,1, \ldots, n-1\}$. (The letters $r_{i}$ denote superscripts, not exponents.)

Applying Lemma 7.5 (ii) repeatedly to each component sequence $\left(j_{u}^{r_{i}}\right)_{i=1}^{\infty}$ and $\left(\tilde{j}_{v}^{r_{i}}\right)_{i=1}^{\infty}$, we obtain after $m_{1}+m_{2}$ steps a subsequence where each component sequence is either constant or strictly increasing.

We have seen in Remark 4.2 that an element $q \in \mathcal{B}_{2}$ may have infinitely many representations, i.e., we may have $q=q_{\mathbf{c}, \mathbf{d}}$ for infinitely many pairs of sequences $(\mathbf{c}, \mathbf{d}) \in \Omega_{q}^{\prime}$. For $q \in \mathcal{B}_{2} \cap\left(1, q_{K L}\right)$ we may have only finitely many representations:

Lemma 7.7. No element $q \in \mathcal{B}_{2} \cap\left(1, q_{K L}\right)$ may be represented by infinitely many pairs of vectors $(\mathbf{k}, \mathbf{s}, \mathbf{j})$ and $(\tilde{\mathbf{k}}, \tilde{\mathbf{s}}, \tilde{\mathbf{j}})$.

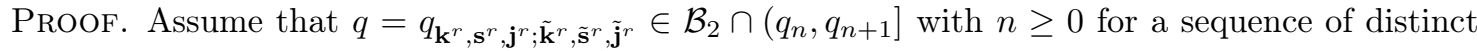
pairs of vectors $\left(\mathbf{k}^{r}, \mathbf{s}^{r}, \mathbf{j}^{r}\right)$ and $\left(\tilde{\mathbf{k}}^{r}, \tilde{\mathbf{s}}^{r}, \tilde{\mathbf{j}}^{r}\right)$. By the preceding lemma we may assume, by taking a subsequence, that each component sequence is either constant or increasing. By Lemma 3.5 each component sequence has to be constant, contradicting the choice of $\left(\mathbf{k}^{r}, \mathbf{s}^{r}, \mathbf{j}^{r}\right)$ and $\left(\tilde{\mathbf{k}}^{r}, \tilde{\mathbf{s}}^{r}, \tilde{\mathbf{j}}^{r}\right)$.

Now we investigate the derived sets $\mathcal{B}_{2}^{(j)}$ in $\left(1, q_{K L}\right)$. In this case we may improve Lemma 6.1 by giving a complete characterization of $\mathcal{B}_{2}^{(j)}$.

Proposition 7.8. Let $\left(q_{n}, q_{n+1}\right)$ be a connected component of $\left(1, q_{K L}\right) \backslash \mathcal{V}$ for some $n \geq 0$, and let $j \geq 0$. A point $q \in \mathcal{B}_{2} \cap\left(q_{n}, q_{n+1}\right]$ belongs to $\left(\mathcal{B}_{2} \cap\left(q_{n}, q_{n+1}\right]\right)^{(j)}$ if and only if $q=q_{\mathbf{k}, \mathbf{s}, \mathbf{j} ; \tilde{\mathbf{k}}, \tilde{\mathbf{s}} \tilde{\mathbf{j}}}$ for some pair of vectors

$$
(\mathbf{k}, \mathbf{s}, \mathbf{j})=\left(k_{1}, \ldots, k_{m_{1}} ; s_{2}, \ldots, s_{m_{1}} ; j_{0}, \cdots, j_{m_{1}-1}, \infty\right)
$$

and

$$
(\tilde{\mathbf{k}}, \tilde{\mathbf{s}}, \tilde{\mathbf{j}})=\left(\tilde{k}_{1}, \ldots, \tilde{k}_{m_{2}} ; \tilde{s}_{2}, \ldots, \tilde{s}_{m_{2}} ; \tilde{j}_{0}, \cdots, \tilde{j}_{m_{2}-1}, \infty\right)
$$

satisfying the condition

$$
m_{1} \geq 0, \quad m_{2} \geq 0 \quad \text { and } \quad 0 \leq\left(k_{m_{1}}+1\right)+\left(\tilde{k}_{m_{2}}+1\right) \leq 2 n-j,
$$

where we write $k_{m_{1}}:=-1$ if $m_{1}=0$ and $\tilde{k}_{m_{2}}:=-1$ if $m_{2}=0$.

Furthermore, $\left(\mathcal{B}_{2} \cap\left(1, q_{n+1}\right]\right)^{(2 n)}=\emptyset$ for all $n \geq 0$. 
Proof. The sufficiency follows from Lemma 6.1. We prove the necessity by induction on $j$.

For $j=0$ the necessity follows from Corollary 7.1 and Lemma 3.2, Now assume that the necessity holds for some $0 \leq j<2 n$, and take $q \in\left(\mathcal{B}_{2} \cap\left(q_{n}, q_{n+1}\right]\right)^{(j+1)}$ arbitrarily. Then there exists a sequence $\left(p_{r}\right) \subset\left(\mathcal{B}_{2} \cap\left(q_{n}, q_{n+1}\right]\right)^{(j)}$ converging to $q$, and we may write

$$
p_{r}=q_{\mathbf{k}^{r}, \mathbf{s}^{r}, \mathbf{j}^{r} ; \tilde{\mathbf{k}}^{r}, \tilde{\mathbf{s}}^{r}, \tilde{\mathbf{j}}^{r}}, \quad r=1,2, \ldots
$$

By Lemma 7.5 we may even assume that the vectors $\mathbf{k}^{r}, \mathbf{s}^{r}, \tilde{\mathbf{k}}^{r}, \tilde{\mathbf{s}}^{r}$ do not depend on $r$, so that we may write simply $\mathbf{k}, \mathbf{s}, \tilde{\mathbf{k}}, \tilde{\mathbf{s}}$, and that each component sequence in $\mathbf{j}^{r}$ and $\tilde{\mathbf{j}}^{r}$ is either constant or strictly increasing. Since the numbers $p_{r}$ are different, at least one of these component sequences is strictly increasing. We may thus write

$$
\left(\mathbf{k}, \mathbf{s}, \mathbf{j}^{r}\right)=\left(k_{1}, \ldots, k_{n_{1}} ; s_{2}, \ldots, s_{n_{1}} ; j_{0}^{r}, \cdots, j_{n_{1}-1}^{r}, \infty\right)
$$

and

$$
\left(\tilde{\mathbf{k}}, \tilde{\mathbf{s}}, \tilde{\mathbf{j}}^{r}\right)=\left(\tilde{k}_{1}, \ldots, \tilde{k}_{n_{2}} ; \tilde{s}_{2}, \ldots, \tilde{s}_{n_{2}} ; \tilde{j}_{0}^{r}, \cdots, \tilde{j}_{n_{2}-1}^{r}, \infty\right)
$$

with

$$
0 \leq\left(k_{n_{1}}+1\right)+\left(\tilde{k}_{n_{2}}+1\right) \leq 2 n-j
$$

by the induction hypothesis. Letting $r \rightarrow \infty$ at least one of the components of $\mathbf{j}^{r}$ or $\tilde{\mathbf{j}}^{r}$ becomes infinite, so that we obtain $q=q_{\mathbf{k}, \mathbf{s}, \mathbf{j} ; \tilde{\mathbf{k}}, \tilde{\mathbf{s}}, \tilde{\mathbf{j}}}$ where

$$
(\mathbf{k}, \mathbf{s}, \mathbf{j})=\left(k_{1}, \ldots, k_{m_{1}} ; s_{2}, \ldots, s_{m_{1}} ; j_{0}, \cdots, j_{m_{1}-1}, \infty\right)
$$

and

$$
(\tilde{\mathbf{k}}, \tilde{\mathbf{s}}, \tilde{\mathbf{j}})=\left(\tilde{k}_{1}, \ldots, \tilde{k}_{m_{2}} ; \tilde{s}_{2}, \ldots, \tilde{s}_{m_{2}} ; \tilde{j}_{0}, \cdots, \tilde{j}_{m_{2}-1}, \infty\right)
$$

with $m_{1}+m_{2}<n_{1}+n_{2}$, and hence

$$
k_{m_{1}}+\tilde{k}_{m_{2}} \leq k_{n_{1}}+\tilde{k}_{n_{2}}-1 \leq 2 n-2-j-1
$$

as claimed.

Note by Theorem 1.1 that $\left(\mathcal{B}_{2} \cap\left(1, q_{1}\right]\right)^{(0)}=\emptyset$. Furthermore, since $\min \mathcal{B}_{2}^{(2)}=q_{2}$ and by Theorem 1.1 that $\mathcal{B}_{2} \cap\left(1, q_{2}\right)=\left\{q_{s}\right\}$, we have $\left(\mathcal{B}_{2} \cap\left(1, q_{2}\right]\right)^{(2)}=\emptyset$. If there exists a $q \in\left(\mathcal{B}_{2} \cap\right.$ $\left.\left(1, q_{n+1}\right]\right)^{(2 n)}$ for some $n \geq 2$, then we infer from (7.1) that $m_{1}=m_{2}=0$, so that $q=q_{\mathbf{k}, \mathbf{s}, \mathbf{j} ; \tilde{\mathbf{k}}, \tilde{\mathbf{s}}, \tilde{\mathbf{j}}}$ with $(\mathbf{k}, \mathbf{s}, \mathbf{j})=(\infty)=(\tilde{\mathbf{k}}, \tilde{\mathbf{s}}, \tilde{\mathbf{j}})$. Then

$$
\left(1 \omega^{\mathbf{k}, \mathbf{s}, \mathbf{j}}\right)_{2}+\left(1 \omega^{\tilde{\mathbf{k}}, \tilde{\mathbf{s}}, \tilde{\mathbf{j}}}\right)_{2}-\left(1^{\infty}\right)_{2}=\left(10^{\infty}\right)_{2}+\left(10^{\infty}\right)_{2}-\left(1^{\infty}\right)_{2}=0
$$

and hence $q=2$, contradicting our assumption $q \in\left(1, q_{n+1}\right]$.

Remark 7.9. In the proof of Proposition 7.8 the selected subsequence $\left(p_{r}\right)$ is monotonic. Indeed, if $j_{n}^{r}$ is the smallest index tending to infinity as $r \rightarrow \infty$, then the corresponding sequence $\left(p_{r}\right)$ is strictly increasing if $s_{n+1}=0$, and strictly decreasing if $s_{n+1}=1$.

Proof of Theorem 1.5 (v) And (vi). (v) was established in Proposition 7.3 .

(vi) Since $\mathcal{B}_{2} \cap\left(1, q_{K L}\right)$ is an infinite set, it suffices to prove the density. Assume on the contrary that there exist an integer $j \geq 0$, a point $q \in \mathcal{B}_{2} \cap\left(1, q_{K L}\right)$ and a closed neighborhood $F$ of $q$ such that $F$ has no isolated points of $\mathcal{B}_{2}$. We may assume that $F \subset\left(1, q_{K L}\right)$. Then all points of the closed set $\mathcal{B}_{2} \cap F$ are accumulation points, so that it is a non-empty perfect set, and hence it is uncountable by a classical theorem of topology. This contradicts the countability of $\mathcal{B}_{2} \cap\left(1, q_{K L}\right)$ by Proposition 7.3 . 
Proof of Theorem 1.7 (v) And (vi). (v) The inequalities follow from Theorem 1.1 (iv) for $j=0$, from Theorem 1.7 (iii) for $j=1$, and from Propositions 6.2 and 7.8 for $j \geq 2$.

Since the sequence $\left(\min \mathcal{B}_{2}^{(j)}\right)$ is non-decreasing by definition, its limit $L$ satisfies the inequalities

$$
q_{K L} \leq L \leq \min \mathcal{B}_{2}^{(\infty)} .
$$

Since $\min \mathcal{B}_{2}^{(\infty)} \leq q_{K L}$ by Theorem 1.7 (i), the claimed limit relations follow.

(vi) We may repeat the proof of Theorem 1.5 (vi) by changing $\mathcal{B}_{2}$ to $\mathcal{B}_{2}^{(j)}$ everywhere.

\section{Local dimension of $\mathcal{B}_{2}$ : proof of Theorem 1.9}

Since $\mathcal{B}_{2} \cap\left(1, q_{K L}\right)$ is at most countable by Proposition 7.3 Theorem 1.9 is trivial for $q<q_{K L}$. Henceforth we assume that $q \in\left[q_{K L}, 2\right]$.

For the proof we recall the following result on Hausdorff dimension and Hölder continuous maps (cf. [13]):

Lemma 8.1. Let $f:\left(X, \rho_{1}\right) \rightarrow\left(Y, \rho_{2}\right)$ be a Hölder map between two metric spaces, i.e., there exist two constants $C>0$ and $\lambda>0$ such that

$$
\rho_{2}(f(x), f(y)) \leq C \rho_{1}(x, y)^{\lambda}
$$

for any $x, y \in X$. Then $\operatorname{dim}_{H} X \geq \lambda \operatorname{dim}_{H} f(X)$.

Given $q \in \mathcal{B}_{2} \cap\left[q_{K L}, 2\right)$ and $\delta \in(0, \min \{q-1,2-q\})$, set

$$
\Omega_{q}^{\prime}(\delta):=\bigcup_{p \in(q-\delta, q+\delta)}\left\{(\mathbf{c}, \mathbf{d}) \in A_{p}^{\prime} \times A_{p}^{\prime}: f_{\mathbf{c}, \mathbf{d}}(p)=0\right\} .
$$

The map

$$
h_{q, \delta}: \Omega_{q}^{\prime}(\delta) \rightarrow \mathcal{B}_{2} \cap(q-\delta, q+\delta), \quad(\mathbf{c}, \mathbf{d}) \mapsto q_{\mathbf{c}, \mathbf{d}}
$$

is well-defined and onto by Lemmas 3.2 and 3.4 Furthermore, by Lemma 3.5 the map $h_{q, \delta}$ is strictly decreasing with respect to each of the vectors $\mathbf{c}$ and $\mathbf{d}$ with $(\mathbf{c}, \mathbf{d}) \in \Omega_{q}^{\prime}(\delta)$.

We recall that the symbolic space $\{0,1\}^{\infty}$ is compact with respect to the metric $\rho$ defined by

$$
\rho\left(\left(c_{i}\right),\left(d_{i}\right)\right):=2^{-\min \left\{j \geq 1: c_{j} \neq d_{j}\right\}} .
$$

In the following lemma we show that the map $h_{q, \delta}$ is also Hölder continuous with respect to the product metric $\rho^{2}$ on $\Omega_{q}^{\prime}(\delta)$ defined by

$$
\rho^{2}((\mathbf{c}, \mathbf{d}),(\tilde{\mathbf{c}}, \tilde{\mathbf{d}})):=\max \{\rho(\mathbf{c}, \tilde{\mathbf{c}}), \rho(\mathbf{d}, \tilde{\mathbf{d}})\}
$$

for any $(\mathbf{c}, \mathbf{d}),(\tilde{\mathbf{c}}, \tilde{\mathbf{d}}) \in \Omega_{q}^{\prime}(\delta)$.

Lemma 8.2. Let $q \in \mathcal{B}_{2} \cap\left[q_{K L}, 2\right)$ and $\delta \in(0,(2-q) / 3)$. Then the function $h_{q, \delta}: \Omega_{q}^{\prime}(\delta) \rightarrow$ $\mathcal{B}_{2} \cap(q-\delta, q+\delta)$ is Hölder continuous of order $\log (q-\delta) / \log 2$ with respect to the product metric $\rho^{2}$ on $\Omega_{q}^{\prime}(\delta)$.

Proof. Fix $q \in \mathcal{B}_{2} \cap\left[q_{K L}, 2\right)$ and $0<\delta<(2-q) / 3$ arbitrarily. Let $(\mathbf{c}, \mathbf{d}),(\tilde{\mathbf{c}}, \tilde{\mathbf{d}}) \in \Omega_{q}^{\prime}(\delta)$, and consider their images $q_{\mathbf{c}, \mathbf{d}}=h_{q, \delta}((\mathbf{c}, \mathbf{d}))$ and $q_{\tilde{\mathbf{c}}, \tilde{\mathbf{d}}}=h_{q, \delta}(\tilde{\mathbf{c}}, \tilde{\mathbf{d}})$ in $\mathcal{B}_{2} \cap(q-\delta, q+\delta)$. Without loss of generality we may assume $\tilde{\mathbf{c}}>\mathbf{c}$ and $\tilde{\mathbf{d}}<\mathbf{d}$. Then there exist positive integers $s$ and $t$ such that

$$
\begin{aligned}
& \tilde{c}_{1} \cdots \tilde{c}_{s-1}=c_{1} \cdots c_{s-1} \quad \text { and } \quad \tilde{c}_{s}>c_{s}, \\
& \tilde{d}_{1} \cdots \tilde{d}_{t-1}=d_{1} \cdots d_{t-1} \quad \text { and } \quad \tilde{d}_{t}<d_{t} .
\end{aligned}
$$


The definitions of $q_{\mathbf{c}, \mathbf{d}}$ and $q_{\tilde{\mathbf{c}}, \tilde{\mathbf{d}}}$ imply that

$$
\left(1^{\infty}\right)_{q_{\mathbf{c}, \mathbf{d}}}=(1 \mathbf{c})_{q_{\mathbf{c}, \mathbf{d}}}+(1 \mathbf{d})_{q_{\mathbf{c}, \mathbf{d}}} \quad \text { and } \quad\left(1^{\infty}\right)_{q_{\tilde{\mathbf{c}}, \tilde{\mathbf{d}}}}=(1 \tilde{\mathbf{c}})_{q_{\tilde{\mathbf{c}}, \tilde{\mathbf{d}}}}+(1 \tilde{\mathbf{d}})_{q_{\tilde{\mathbf{c}}, \tilde{\mathbf{d}}}} .
$$

Hence

$$
\begin{aligned}
\left|\frac{1}{q_{\mathbf{c}, \mathbf{d}}-1}-\frac{1}{q_{\tilde{\mathbf{c}}, \tilde{\mathbf{d}}}-1}\right| & =\left|(1 \mathbf{c})_{q_{\mathbf{c}, \mathbf{d}}}+(1 \mathbf{d})_{q_{\mathbf{c}, \mathbf{d}}}-(1 \tilde{\mathbf{c}})_{q_{\tilde{\mathbf{c}}, \tilde{\mathbf{d}}}}-(1 \tilde{\mathbf{d}})_{q_{\tilde{\mathbf{c}}, \tilde{\mathbf{d}}}}\right| \\
& \leq\left|(1 \mathbf{c})_{q_{\mathbf{c}, \mathbf{d}}}-(1 \tilde{\mathbf{c}})_{q_{\tilde{\mathbf{c}}, \tilde{\mathbf{d}}}}\right|+\left|(1 \mathbf{d})_{q_{\mathbf{c}, \mathbf{d}}}-(1 \tilde{\mathbf{d}})_{q_{\tilde{\mathbf{c}}, \tilde{\mathbf{d}}}}\right|
\end{aligned}
$$

Since $q_{\mathbf{c}, \mathbf{d}}, q_{\tilde{\mathbf{c}}, \tilde{\mathbf{d}}}>q-\delta$, we have

$$
\begin{aligned}
\left|(1 \mathbf{c})_{q_{\mathbf{c}, \mathbf{d}}}-(1 \tilde{\mathbf{c}})_{q_{\tilde{\mathbf{c}}, \tilde{\mathbf{d}}} \mid}\right|= & \left(1 c_{1} \cdots c_{s-1} 0^{\infty}\right)_{q_{\mathbf{c}, \mathbf{d}}}-\left(1 c_{1} \cdots c_{s-1} 0^{\infty}\right)_{q_{\tilde{\mathbf{c}}, \tilde{\mathbf{d}}}} \\
& \quad+\left(0^{s} c_{s} c_{s+1} \cdots\right)_{q_{\mathbf{c}, \mathbf{d}}}-\left(0^{s} \tilde{c}_{s} \tilde{c}_{s+1} \cdots\right)_{q_{\tilde{\mathbf{c}}, \tilde{\mathbf{d}}}} \mid \\
\leq & \left|\left(20^{\infty}\right)_{q_{\mathbf{c}, \mathbf{d}}}-\left(20^{\infty}\right)_{q_{\tilde{\mathbf{c}}, \tilde{\mathbf{d}}}}\right| \\
& \quad+\left|\left(0^{s} 1^{\infty}\right)_{q_{\mathbf{c}, \mathbf{d}}}\right|+\left|\left(0^{s} 1^{\infty}\right)_{q_{\tilde{\mathbf{c}}, \tilde{\mathbf{d}}}}\right| \\
\leq & \frac{2\left|q_{\tilde{\mathbf{c}}, \tilde{\mathbf{d}}}-q_{\mathbf{c}, \mathbf{d}}\right|}{(q-\delta)^{2}}+\frac{2}{(q-\delta)^{s}(q-\delta-1)}
\end{aligned}
$$

Similarly, we also have

$$
\left|(1 \mathbf{d})_{q_{\mathbf{c}, \mathbf{d}}}-(1 \tilde{\mathbf{d}})_{q_{\tilde{\mathbf{c}}, \tilde{\mathbf{d}}}}\right| \leq \frac{2\left|q_{\tilde{\mathbf{c}}, \tilde{\mathbf{d}}}-q_{\mathbf{c}, \mathbf{d}}\right|}{(q-\delta)^{2}}+\frac{2}{(q-\delta)^{t}(q-\delta-1)} .
$$

Therefore, using (8.1) it follows that

$$
\begin{aligned}
\left|\frac{1}{q_{\mathbf{c}, \mathbf{d}}-1}-\frac{1}{q_{\tilde{\mathbf{c}}, \tilde{\mathbf{d}}}-1}\right| & \leq \frac{4\left|q_{\tilde{\mathbf{c}}, \tilde{\mathbf{d}}}-q_{\mathbf{c}, \mathbf{d}}\right|}{(q-\delta)^{2}}+\frac{2}{q-\delta-1}\left(\frac{1}{(q-\delta)^{s}}+\frac{1}{(q-\delta)^{t}}\right) \\
& =\frac{4\left|q_{\tilde{\mathbf{c}}, \tilde{\mathbf{d}}}-q_{\mathbf{c}, \mathbf{d}}\right|}{(q-\delta)^{2}}+\frac{2}{q-\delta-1}\left(2^{\left.-s^{\frac{\log (q-\delta)}{\log 2}}+2^{-t \frac{\log (q-\delta)}{\log 2}}\right)}\right. \\
& \leq \frac{4\left|q_{\tilde{\mathbf{c}}, \tilde{\mathbf{d}}}-q_{\mathbf{c}, \mathbf{d}}\right|}{(q-\delta)^{2}}+\frac{4}{q-\delta-1}\left(\rho^{2}((\mathbf{c}, \mathbf{d}),(\tilde{\mathbf{c}}, \tilde{\mathbf{d}}))\right)^{\frac{\log (q-\delta)}{\log 2}}
\end{aligned}
$$

On the other hand, since $q_{\mathbf{c}, \mathbf{d}}, q_{\tilde{\mathbf{c}}, \tilde{\mathbf{d}}}<q+\delta$, we have

$$
\left|\frac{1}{q_{\mathbf{c}, \mathbf{d}}-1}-\frac{1}{q_{\tilde{\mathbf{c}}, \tilde{\mathbf{d}}}-1}\right| \geq \frac{\left|q_{\tilde{\mathbf{c}}, \tilde{\mathbf{d}}}-q_{\mathbf{c}, \mathbf{d}}\right|}{(q+\delta-1)^{2}} .
$$

Comining with (8.2) we conclude that

$$
\left|q_{\tilde{\mathbf{c}}, \tilde{\mathbf{d}}}-q_{\mathbf{c}, \mathbf{d}}\right| \leq\left(\frac{1}{(q+\delta-1)^{2}}-\frac{4}{(q-\delta)^{2}}\right)^{-1} \frac{4}{q-\delta-1}\left(\rho^{2}((\mathbf{c}, \mathbf{d}),(\tilde{\mathbf{c}}, \tilde{\mathbf{d}}))\right)^{\frac{\log (q-\delta)}{\log 2}},
$$

where the fractional term on the right hand side is positive since $\delta<(2-q) / 3$ and $q-\delta-1>0$.

Proof of Theorem 1.9. Let $q \in \mathcal{B}_{2} \cap\left[q_{K L}, 2\right)$ and $\delta \in(0,(2-q) / 3)$. Using Lemmas 8.1 and 8.2 we get

$$
\operatorname{dim}_{H}\left(\mathcal{B}_{2} \cap(q-\delta, q+\delta)\right) \leq \frac{\log 2}{\log (q-\delta)} \operatorname{dim}_{H} \Omega_{q}^{\prime}(\delta) \leq \frac{\log 2}{\log (q-\delta)} \operatorname{dim}_{H}\left(\mathcal{U}_{q+\delta}^{\prime} \times \mathcal{U}_{q+\delta}^{\prime}\right),
$$


where the last inequality follows because $\Omega_{q}^{\prime}(\delta)$ is a subset of $\mathcal{U}_{q+\delta}^{\prime} \times \mathcal{U}_{q+\delta}^{\prime}$. Observe that $\left(\mathcal{U}_{q+\delta}^{\prime}, \rho\right)$ is a fractal set whose Hausdorff dimension is given by

$$
\operatorname{dim}_{H} \mathcal{U}_{q+\delta}^{\prime}=\frac{h_{t o p}\left(\mathcal{U}_{q+\delta}^{\prime}\right)}{\log 2} .
$$

Hence, using (8.3) we obtain that

$$
\begin{aligned}
\operatorname{dim}_{H}\left(\mathcal{B}_{2} \cap(q-\delta, q+\delta)\right) & \leq \frac{\log 2}{\log (q-\delta)} \operatorname{dim}_{H}\left(\mathcal{U}_{q+\delta}^{\prime} \times \mathcal{U}_{q+\delta}^{\prime}\right) \\
& \leq \frac{2 \log 2}{\log (q-\delta)} \operatorname{dim}_{H} \mathcal{U}_{q+\delta}^{\prime}=2 \frac{h_{t o p}\left(\mathcal{U}_{q+\delta}^{\prime}\right)}{\log (q-\delta)} .
\end{aligned}
$$

Letting $\delta \rightarrow 0$ and applying Lemma 2.4 we conclude that

$$
\lim _{\delta \rightarrow 0} \operatorname{dim}_{H}\left(\mathcal{B}_{2} \cap(q-\delta, q+\delta)\right) \leq 2 \frac{h_{\text {top }}\left(\mathcal{U}_{q}^{\prime}\right)}{\log q}=2 \operatorname{dim}_{H} \mathcal{U}_{q}
$$

\section{Final remarks}

In this section we present some open questions.

We have seen that $\mathcal{B}_{2}$ has infinitely many isolated points in $\left(1, q_{K L}\right)$.

Question 1. Does $\mathcal{B}_{2}$ have any isolated points greater than $q_{K L}$ ?

By Theorems 1.1 and 1.9 there exists a smallest number $q_{*} \in\left(q_{K L}, \varphi_{3}\right]$ such that $\left[q_{*}, 2\right] \subset \mathcal{B}_{2}$.

Question 2. What is this smallest number $q_{*}$ ?

Sidorov proved in [27] that $q_{s} \approx 1.71064$ is an accumulation point of $\mathcal{B}_{\aleph_{0}}$, and then Baker proved in [2] that $q_{s}$ is in fact its smallest accumulation point.

We have shown in Theorem 1.5 that $q_{f} \approx 1.75488$ is the smallest accumulation point of $\mathcal{B}_{2}$ and of $\mathcal{B}_{2}^{(1)}$.

Question 3. What is the smallest element of $\mathcal{B}_{2}^{(j)}$ for $j=3,4, \ldots$ ?

Question 4. What is the smallest accumulation point of $\mathcal{B}_{k}$ for $k=3,4, \ldots$ ? (This question has already been raised in [3].)

We have shown in Theorem 1.5 that $\mathcal{B}_{2}$ is closed, and it was proved in [29] by Zou and Kong that $\mathcal{B}_{\aleph_{0}}$ is not closed.

Question 5. Are the sets $\mathcal{B}_{k}$ closed or not for $k=3,4, \ldots$ ?

Recently, the second author and his coauthors studied in [23] the smallest element of $\mathcal{B}_{2}$ for multiple digit sets $\{0,1, \ldots, m\}$. The ideas and methods in this paper might also be useful to further explore $\mathcal{B}_{2}$ in the multiple digit set case. For this extension the main difficulty we may encounter is the characterization of $\mathcal{B}_{2}$ as in Section 3. Furthermore, a generalization of Theorem 5.3 for the explicit description of $\mathcal{U}_{q_{0}^{*}}^{\prime} \backslash \mathcal{U}_{q_{0}}^{\prime}$ is also needed.

Each $q \in \overline{\mathcal{U}} \backslash \mathcal{U} \subset \mathcal{B}_{2}$ has infinitely many representations by Remark 4.2 , and this cannot happen for $q \in \mathcal{B}_{2} \cap\left(1, q_{K L}\right)$ by Lemma 7.7 .

In the last three questions we consider the elements of $\mathcal{B}_{2} \cap\left(1, q_{K L}\right)$.

Question 6. Is the representation of each $q \in \mathcal{B}_{2} \cap\left(1, q_{K L}\right)$ unique?

Question 7. Is it true that $q_{n+1}$ is isolated from the left in $\mathcal{B}_{2}$ ?

Question 8. Is it possible that $q_{n+1} \in \mathcal{B}_{2}^{(3)}$ for some $n$ ? 


\section{Acknowledgements}

The authors thank the anonymous referee for many useful suggestions. The second author was supported by NSFC No. 11401516. He would like to thank Professor Yann Bugeaud for his hospitality when he visited Strasbourg University in November, 2016.

\section{References}

\section{References}

[1] C. Baiocchi, V. Komornik, Greedy and quasi-greedy expansions in non-integer bases, arXiv:0710.3001v1.

[2] S. Baker, On small bases which admit countably many expansions, J. Number Theory 147 (2015) 515-532.

[3] S. Baker, N. Sidorov, Expansions in non-integer bases: lower order revisited, Integers 14 (2014) Paper No. A57, 1-15.

[4] C. Bonanno, C. Carminati, S. Isola, G. Tiozzo, Dynamics of continued fractions and kneading sequences of unimodal maps, Discrete Contin. Dyn. Syst. 33 (4) (2013) 1313-1332.

[5] K. Dajani, M. de Vries, Invariant densities for random $\beta$-expansions, J. Eur. Math. Soc. (JEMS) 9 (1) (2007) 157-176.

[6] Z. Daróczy, I. Kátai, On the structure of univoque numbers, Publ. Math. Debrecen 46 (3-4) (1995) 385-408.

[7] M. de Vries, V. Komornik, Unique expansions of real numbers, Adv. Math. 221 (2) (2009) 390-427.

[8] M. de Vries, V. Komornik, Expansions in non-integer bases, Combinatorics, words and symbolic dynamics, Encyclopedia Math. Appl., vol. 159, Cambridge Univ. Press, Cambridge, 2016, pp. 18-58.

[9] M. de Vries, V. Komornik, P. Loreti, Topology of the set of univoque bases, Topology Appl. 205 (2016) 117-137.

[10] P. Erdôs, I. Joó, V. Komornik, Characterization of the unique expansions $1=\sum_{i=1}^{\infty} q^{-n_{i}}$ and related problems, Bull. Soc. Math. France 118 (1990) 377-390.

[11] P. Erdős, M. Horváth, I. Joó, On the uniqueness of the expansions $1=\sum q^{-n_{i}}$, Acta Math. Hungar. 59 (3-4) (1991) 333-342.

[12] P. Erdôs, I. Joó, On the number of expansions $1=\sum q^{-n_{i}}$, Ann. Univ. Sci. Budapest. Eötvös Sect. Math. 35 (1992) 129-132.

[13] K. Falconer, Fractal geometry, John Wiley \& Sons Ltd., Chichester, 1990, Mathematical foundations and applications.

[14] P. Glendinning and N. Sidorov, Unique representations of real numbers in non-integer bases, Math. Res. Lett. 8 (2001) 535-543.

[15] V. Komornik, Expansions in noninteger bases, Integers 11B (2011) Paper No. A9, 1-30.

[16] V. Komornik, Unique infinite expansions in noninteger bases, Acta Math. Hungar. 134 (3) (2012) 344-355. 
[17] V. Komornik, Lectures on functional analysis and the Lebesgue integral, Universitext, Springer-Verlag, London, 2016, Translated from the 2002 French original.

[18] V. Komornik, D. Kong, W. Li, Hausdorff dimension of univoque sets and devil's staircase, Adv. Math. 305 (2017) 165-196.

[19] V. Komornik, P. Loreti, Unique developments in non-integer bases, Amer. Math. Monthly 105 (7) (1998) 636-639.

[20] V. Komornik, P. Loreti, On the topological structure of univoque sets, J. Number Theory 122 (1) (2007) 157-183.

[21] D. Kong, W. Li, Hausdorff dimension of unique beta expansions, Nonlinearity 28 (1) (2015) 187-209.

[22] D. Kong, W. Li, F. Lü, M. de Vries, Univoque bases and hausdorff dimension, Monatsh. Math. 184 (3) (2017) 443-458.

[23] D. Kong, W. Li, Y. Zou, On small bases which admit points with two expansions, J. Number Theory 173 (2017) 100-128.

[24] J. Kürschák, Analízis és analitikus geometria [Analysis and analytic geometry], Budapest, 1920.

[25] W. Parry, On the $\beta$-expansions of real numbers, Acta Math. Acad. Sci. Hungar. 11 (1960) 401-416.

[26] N. Sidorov, Almost every number has a continuum of $\beta$-expansions, Amer. Math. Monthly 110 (9) (2003) 838-842.

[27] N. Sidorov, Expansions in non-integer bases: lower, middle and top orders, J. Number Theory 129 (4) (2009) 741-754.

[28] W. Steiner, Review of the paper [16], Mathematical Reviews, MR2886211.

[29] Y. Zou, D. Kong, On a problem of countable expansions, J. Number Theory 158 (2016) 134-150. 